\title{
QUEEN'S
UNIVERSITY
BELFAST
}

\section{Questions in Livestock Helminthology Research}

Morgan, E., Aziz, Blanchard, Charlier, Charvet, Claerebout, Geldhof, Greer, Hertzberg, Hodgkinson, Hoglund, Hoste, Kaplan, Martinez-Valladares, Mitchell, Ploeger, Rinaldi, van Samson-Himmelstjerna, Sotiraki, ... Vercruysse, J. (2018). 100 Questions in Livestock Helminthology Research. Trends in Parasitology. https://doi.org/10.1016/j.pt.2018.10.006

\section{Published in:}

Trends in Parasitology

\section{Document Version:}

Peer reviewed version

\section{Queen's University Belfast - Research Portal:}

Link to publication record in Queen's University Belfast Research Portal

\section{Publisher rights}

Copyright 2018 Elsevier

This manuscript is distributed under a Creative Commons Attribution-NonCommercial-NoDerivs License

(https://creativecommons.org/licenses/by-nc-nd/4.0/), which permits distribution and reproduction for non-commercial purposes, provided the author and source are cited.

\section{General rights}

Copyright for the publications made accessible via the Queen's University Belfast Research Portal is retained by the author(s) and / or other copyright owners and it is a condition of accessing these publications that users recognise and abide by the legal requirements associated with these rights.

\section{Take down policy}

The Research Portal is Queen's institutional repository that provides access to Queen's research output. Every effort has been made to ensure that content in the Research Portal does not infringe any person's rights, or applicable UK laws. If you discover content in the Research Portal that you believe breaches copyright or violates any law, please contact openaccess@qub.ac.uk. 


\section{Questions in Livestock Helminthology Research}

2 Eric R. Morgan ${ }^{1 *}$, Nor-Azlina A. Aziz $^{2}$, Alexandra Blanchard ${ }^{3}$, Johannes Charlier ${ }^{4}$,

3 Claude Charvet $^{5}$, Edwin Claerebout ${ }^{6}$, Peter Geldhof ${ }^{6}$, Andrew W. Greer ${ }^{7}$, Hubertus

4 Hertzberg ${ }^{8}$, Jane Hodgkinson ${ }^{9}$, Johan Höglund ${ }^{10}$, Hervé Hoste ${ }^{11}$, Ray M. Kaplan ${ }^{12}$

5 María Martínez Valladares ${ }^{13}$, Siân Mitchell ${ }^{14}$, Harm W. Ploeger ${ }^{15}$, Laura Rinaldi ${ }^{16}$,

6 Georg von Samson-Himmelstjerna ${ }^{17}$, Smaragda Sotiraki ${ }^{18}$, Manuela Schnyder ${ }^{8}$, Philip

7 Skuce $^{19}$, David Bartley ${ }^{19}$, Fiona Kenyon ${ }^{19}$, Stig M. Thamsborg ${ }^{20}$, Hannah Rose Vineer ${ }^{21}$,

8 Theo de Waal $^{22}$, Andrew R. Williams ${ }^{20}$, Jan A. van Wyk ${ }^{23}$, Jozef Vercruysse ${ }^{6}$

9

1. Queen's University Belfast, School of Biological Sciences, 97, Lisburn Road, Belfast, BT9

$117 \mathrm{BL}$, Northern Ireland, United Kingdom.

2. Department of Veterinary Pathology and Microbiology, Faculty of Veterinary Medicine, Universiti Putra Malaysia, 43400 UPM, Serdang, Selangor, Malaysia.

3. Pancosma, voie des traz 6, CH-1218 Le Grand Saconnex (Geneva), Switzerland.

4. Kreavet, Hendrik Mertensstraat 17, 9150 Kruibeke, Belgium.

5. ISP, INRA, Université Tours, UMR1282, 37380, Nouzilly, France.

6. Laboratory for Parasitology, Faculty of Veterinary Medicine, Ghent University, B9820 Merelbeke, Belgium.

7. Faculty of Agriculture and Life Sciences, P.O. Box 85084, Lincoln University, Christchurch, 7647, New Zealand.

8. Institute of Parasitology, University of Zurich, Winterthurerstrasse 266a, 8057 Zurich, Switzerland.

9. Institute of Infection and Global Health, University of Liverpool, Liverpool Science Park 
10. Swedish University of Agricultural Sciences, BVF-parasitology, Box 7036, 75007 , Uppsala, Sweden.

11. UMR 1225 IHAP INRA/ENVT, 23 Chemin des Capelles, 31076 Toulouse, France.

12. Department of Infectious Diseases, College of Veterinary Medicine, University of Georgia, Athens, Georgia, USA.

13. Instituto de Ganadería de Montaña (CSIC-Universidad de León). Finca Marzanas, Grulleros, 24346 León, Spain.

14. Animal and Plant Health Agency, Carmarthen Veterinary Investigation Centre, Jobswell Rd, Johnstown, Carmarthen, SA31 3EZ, Wales, United Kingdom.

15. Utrecht University, Department of Infectious Diseases and Immunology, Yalelaan 1, 3584 CL, Utrecht, The Netherlands.

16. Department of Veterinary Medicine and Animal Production, University of Napoli Federico II, Napoli, Italy.

17. Institute for Parasitology and Tropical Veterinary Medicine, Freie Universitaet Berlin, Robert-von-Ostertag-Str. 7-13, 14163 Berlin, Germany.

18.Veterinary Research Institute, HAO-DEMETER, Campus Thermi 57001 Thessaloniki Greece.

19. Moredun Research Institute, Pentlands Science Park, Edinburgh EH26 0PZ, Scotland, United Kingdom.

20. Department of Veterinary and Animal Sciences, Faculty of Health and Medical Sciences, University of Copenhagen, Frederiksberg, Denmark.

21. School of Biological Sciences, University of Bristol, 24 Tyndall Avenue, Bristol, BS8 1TQ, United Kingdom.

22. University College Dublin, School of Veterinary Medicine, Belfield, Dublin, D04 W6F6, Ireland. 
23. Department of Veterinary Tropical Diseases, University of Pretoria, Private Bag X20, Pretoria, South Africa.

*Correspondence: eric.morgan@qub.ac.uk

54 Abstract

55 An elicitation exercise was conducted to collect and identify pressing questions concerning

56 the study of helminths in livestock, to help guide research priorities. Questions were invited

57 from the research community in an inclusive way. Of 385 questions submitted, 100 were

58 chosen by online vote, with priority given to open questions in important areas that are

59 specific enough to permit investigation within a focused project or programme of research.

60 The final list of questions was divided into ten themes. We present the questions and set them

61 briefly in the context of the current state of knowledge. Although subjective, results provide a

62 snapshot of current concerns and perceived priorities in the field of livestock helminthology,

63 and we hope will stimulate ongoing or new research efforts.

\section{Key words:}

65 Helminth parasite, nematode, trematode, livestock, anthelmintic resistance, research priorities 
The study of the helminth parasites of livestock is facing a period of rapid change. The availability of a series of highly effective and affordable anthelmintics from the 1960s onwards coincided with the intensification of animal production systems in many parts of the world. As a result, adequate control of helminths could be achieved on the majority of farms with existing scientific knowledge, reducing incentives for investment in further research [1]. Currently, however, the effectiveness of control is breaking down in various areas. Anthelmintic resistance (AR) is increasing worldwide in helminths of all livestock species, highlighting the reliance of modern food production on chemical control of pests and parasites, and threatening the sustainability of livestock production, especially in grazing systems [2-4]. At the same time, changes in weather and climate are making infection patterns less predictable, and fixed protocol-driven approaches to helminth control are consequently less reliable [5]. To counter these challenges, alternative methods for helminth control are being developed, including, for example, vaccines, biological control, bioactive forages, grazing management, selective breeding, and various ways of targeting treatment in response to indicators of parasite infection or its impacts [6]. Development and effective application of novel control approaches require a return to fundamental scientific research to underpin future advances in parasite management. This renaissance of interest in veterinary helminthology comes at a time when it might profitably harness an explosion of new technologies, arising from rapid advances in molecular biology and 'omics', predictive modelling and data mining, sensor technologies and other fields [1]. In order to address research challenges and opportunities in relation to animal diseases, including those caused by helminths in livestock, new formal groupings serve to augment existing collaborations and provide a platform for coordination, mainly at European level

91 (Box 1). In some, experts are enlisted in structured gap analyses to stimulate research and 
92 feed into priority-setting by funders and policy makers, as well as produce published outputs

$93[7,8]$. In other cases, experts produce opinionated reviews on the state of the art and expound

94 a vision of the way forward [1,4,9]. These exercises are built on consensus, often among

95 those who have worked together over a sustained period to develop ideas and drive progress

96 in the field. While these approaches are undoubtedly useful, they tend to perpetuate dominant

97 current thinking, and potentially neglect marginal but promising suggestions.

98 Alternatives are possible. Inspired by previous attempts in ecology [10], we here consult

99 more widely across the research community to identify key current questions in livestock

100 helminthology, to motivate and guide new work. The number 100 was chosen such that

101 questions might be broad enough to be strategically important, yet focused enough to be

102 tackled within a single focused research project or programme [10]. We elicited questions

103 from as wide a base as possible within the discipline (Box 2), to reduce the influence of

104 expert views and established dogmas on the questions presented, and to allow for disruptive

105 and creative ideas. Further rounds of voting and organization followed, and here we list the

106 questions judged most meritorious by a broad panel of specialists. The ten sub-sections are

107 based on the questions received and were not decided beforehand, and text commentary

108 follows rather than precedes each series of questions, in keeping with the 'bottom-up' spirit

109 of the exercise. The sections are structured to progress in a general direction from processes

110 of infection, through impacts, to control through chemical and alternative means, and include

111 challenges across the spectrum of fundamental and applied research. While we make no

112 claim to this list being definitive or complete, it is a snapshot of what researchers in livestock

113 helminthology consider to be important and topical at this time, and we hope that it will

114 stimulate discussion, and renew energy in existing or novel directions.

\section{Section I: Helminth biology and epidemiology}


118 1. What determines emergence of arrested helminth stages in the host, e.g. termination of

119 hypobiosis in gastrointestinal nematodes in ruminants or cyathostomins in horses, or end of

120 the mucosal phase of ascarids in poultry?

121

122 Hypobiosis is important for perpetuation of helminth populations during adverse environmental conditions. While factors inducing hypobiosis are well described (e.g. cold or dry seasonal cues, or immunity), factors governing the period of inhibition and timing of emergence are poorly understood. Intrinsic parasite factors, host physiology, or seasonality may all play a role $[11,12]$, but the biochemical basis for these is mostly unknown. New molecular methods, e.g. transcriptomics, may be useful to understand mechanisms of emergence from arrest [13]. Resulting knowledge may pave the way for new control options during a phase when the therapeutic arsenal is typically limited due to the very low metabolic activity of the hypobiotic stages.

\section{$132 \quad$ Fecundity}

133 2. What regulates egg production in female helminths and can it be suppressed sufficiently to 134 provide an epidemiological advantage?

135 3. Will breeding for host resistance (low faecal egg counts) drive nematode adaptation 136 towards increased fecundity to compensate?

138 Interference with female worm fecundity could contribute to helminth control, and would

139 benefit from detailed mapping of influencing factors, like host dietary, physiological and

140 immunological status, location in the host, and intrinsic parasite factors, e.g. genetic

141 predisposition and environment-induced changes. For example, in Haemonchus contortus, 
142 worm size is highly correlated with the number of eggs present in adult females, and egg

143 production is limited by host immune regulation [15]. Ability to target fecundity specifically,

144 and evolutionary responses of parasites to such a strategy, are therefore likely to be highly

145 dependent on other parasite traits as well as host factors.

147 Parasite adaptation to new hosts

148 4. To what extent is there an exchange of parasites between wild and domestic ruminants?

149 5. Does cross-grazing of cattle and small ruminants encourage gastrointestinal nematode

150 species to adapt and cross between hosts?

152 Gastrointestinal nematode (GIN) species tend to have a preferred host, but there is considerable evidence to indicate transmission and adaptation between livestock species

154 (sheep/goat/cattle) and between livestock and wildlife when either co-grazed or grazed

155 alternately on the same pasture [15]. In farming systems, control by means of alternate

156 grazing with different host species has been reported to break down due to parasite adaptation

157 [16]. Older studies often lack genotyping and apparent infection across multiple host species may therefore constitute different parasite subpopulations or even species with cryptic host preferences, as with lungworms in deer [17]. Whether the impact of cross-transmission

160 between wildlife and livestock is likely to amplify or reduce pasture infectivity and thus

161 transmission to livestock is in general an open question and likely to be context-specific [18].

162 Untreated wildlife could, moreover, act as a source of refugia for drug-susceptible genotypes, 163 or alternatively transfer resistant parasites to new hosts or locations [19]. The net effect of

164 livestock-wildlife contact on helminth ecology and evolution is hard to predict. 
167 6. How do parasitic worms respond to climatic change and what is their environmental 168 plasticity?

169 7. What is the effect of climate and weather, especially drought, on the spatial distribution of

170 infective helminth larvae on pasture and on the subsequent risk for grazing animals?

1718 . How is climate change affecting overwintering of nematodes in temperate areas?

172 9. Will climate change result in a change of helminth species in temperate environments or 173 will the existing ones simply adapt?

174 10. Is the recent increase in the prevalence of rumen fluke in Europe a threat to livestock 175 farming?

177 Climate changes may not only affect helminths directly (e.g. the external stages and induction of hypobiosis) but also via effects on availability of definitive or intermediate hosts or on habitats, and through land use in agriculture. In general, parasites tend to adapt to the changes happening around them by evolving. Adaptation may involve strain variation in phenology,

181 within-genotype variation in key life history traits and host switching [20]. Parasites may 182 spread their chances of infecting hosts across variable or changing environments. An example in livestock is the adaptive epidemiology of Nematodirus battus, previously having a single generation per year (spring infection), but more recently evolving a strategy of two generations per year, which is better suited to unpredictable spring weather [21]. Parallel

186 work on microbes indicates that sensitivity to environmental variation is itself a trait that can

187 evolve, conferring resilience to changing climates [22]. There is considerable scope to improve predictions and measurements of helminth responses to climate change, in terms of evolutionary as well as epidemiological dynamics, and to include helminths with indirect life cycles such as trematodes, in which adaptive changes in intermediate hosts might also be

191 important. Differentiating climate change from other forces and proving its role in parasite 
192 range expansion is not straightforward, either for apparently emerging parasites such as the rumen fluke Calicophoron daubneyi [23] or for other helminths, and this undermines attempts to predict future challenges to farming. Given the multiple interacting factors that drive parasite epidemiology, research should embed parasitic disease in wider studies of climate change mitigation and adaptation in livestock and mixed agricultural systems [24].

Improved diagnostics for epidemiological monitoring

11. Can we develop good ways to enumerate infective helminth stages on pasture?

Various methods have been extensively documented to recover infective stages of GINs and flukes from herbage or tracer animals, followed by microscopic counting and identification by morphological or molecular methods [25]. However, modern quantitative and qualitative molecular methods have not been sufficiently adapted for rapid estimation of the level of parasite challenge. Success would have clear applications to parasite management as well as improving the feasibility of field studies to test epidemiological and evolutionary predictions.

\section{Section II: Economic and environmental impacts}

12. What is the true financial cost of helminth infection?

13. Is profitable livestock husbandry possible without chemical parasite control?

211 14. Does the control of helminths reduce net methane emission over the lifetime of a

212 ruminant?

213 15. How can environmental impacts of anthelmintics be properly measured, including on

214 non-target fauna, and ecosystem functioning and service provision?

215 16. What are the costs (financial, human and to animal welfare) of anthelmintic resistance? 
218 The established aim of helminth control is to reduce parasite burden to improve animal health

219 and productivity. As a result, research has tended to focus on how novel parasite control

220 approaches can achieve higher efficacy and optimise production. Today, increasing emphasis

221 is being placed on the sustainability of livestock farming. Therefore, the use of all inputs

222 needs to be accounted for in the production equation and the role of helminth infection needs

223 to be clarified in terms of optimal farm resource allocation, as well as its environmental and

224 economic impacts [26]. There is early evidence from experimental and field studies of the

225 beneficial impacts of effective helminth control on reducing greenhouse gas emission

226 intensity in grazing livestock [27-29]. The impact of helminth parasitism on water use

227 efficiency also needs to be better understood. There is a need to extend these approaches to emerging and resurgent parasite species such as rumen fluke and to investigate the direct impacts of failure of control, for example as a result of anthelmintic resistance.

\section{Costing environmental impacts of drugs and drug resistance}

232 Side-effects of anthelmintics as a consequence of 'leakage' into the environment, such as on non-target fauna [30] and onward impacts on their ecology and ecosystem service provision

234 [31] need to be better understood and balanced against the beneficial impacts of treatment. The direct costs of anthelmintic resistance include the cost of the ineffective drug,

236 the labour wastage in administering the ineffective drug, and the failure of adequate control

237 leading to reduced production of meat and milk on a per hectare and per animal basis.

238 However, there likely are many other indirect economic and environmental impacts since

239 more animals will be needed to produce the same amount of food [32]. Generating these

240 insights and integrating them into economic frameworks has great potential to support

241 sustainable helminth control programmes at farm, regional and national levels. Valuing 
sustainability, and the economic benefits of helminth control in less monetised farming systems, remain challenging [33].

\section{Section III: Effects on host behaviour and welfare}

17. How can we measure the impact of helminth infections on livestock welfare?

18. How does parasitism affect animal behaviour?

19. Can we use changes in behaviour to identify those individuals that need treatment?

20. Can we select for host behaviour to control helminths?

21. Do ruminants self-medicate by selectively grazing plants with anthelmintic compounds?

22. Are animals better off and healthier with some worms, rather than none? Studies are biased towards negative effects on hosts, and neglect potentially positive outcomes at individual and population levels.

\section{Measuring behavioural impacts of parasitism}

256 Research into the impacts of helminth infections on the behaviour and welfare of livestock has largely focused on aspects of direct economic importance in ruminant livestock [34], and is lagging behind research into the behavioural and welfare impacts of parasites in other hostparasite systems [35]. The impact of subclinical helminth infection on host behaviour and welfare indicators remains largely understudied, perhaps in part because such subclinical

261 effects can be hard to detect and difficult to separate from those of other disorders. Still,

262 changes can be more objectively measured today using new technologies. Thus, advances in electronic technology (e.g. 3D accelerometers), offers novel tools to monitor and detect host welfare and behavioural responses to parasitism and to link these to targeted control efforts [36]. Further, positive behaviours that allow livestock to avoid or suppress infection, such as

266 self-medication and selective grazing, may be identified as markers to selectively breed for 
'behavioural' resistance [37]. The importance of behaviour as a defence strategy against GIN is recognized in goats [38], but empirical evidence for selectively breeding grazing animals to develop this trait is so far lacking.

270

Helminth infection is not necessarily negative

272 Studies to date focus on negative effects on hosts, and neglect potentially positive outcomes of helminth infections, such as regulatory roles at scales ranging from gut microbiomes and inflammation [39] to entire grazing systems [40]. Studies taking a more holistic view of the consequences of infection for individual and group health would be timely given changes in

276 farming systems and increasing societal concern in many countries for the welfare and environmental costs of modern farming practices.

278

\section{Section IV: Host-helminth-microbiome interactions}

23. How do gastrointestinal parasites communicate in the gut?

24. How does interaction between different helminths in co-infection affect the immune system of the host and the development of disease?

25. Are there associations between animals' microbiomes and helminth communities, and do they matter?

26. Can the alteration of gut microbiota influence immunity to parasites in livestock, and vice

286 versa?

287 27. To what extent do co-infections between helminths and other specific pathogens, e.g.

288 liver fluke and bovine tuberculosis; gastrointestinal nematodes and paratuberculosis;

289 lungworms and respiratory pathogens; influence health outcomes for livestock and human 290 health? 
293 The ability of helminths to influence the host response and dictate disease outcomes of co-

294 infections is an active area of research within parasitology [41], in which many questions

295 remain unanswered. In classical co-infection scenarios, a co-evolutionary dynamic between

296 the vertebrate host, helminths and microbiome is thought to result from complex adaptations

297 of each of the three components [42]. Research into helminth-microbiota co-infections in

298 livestock hosts is in its early stages, raising questions about whether a host's microbiome and

299 helminth community interact and communicate, how any such interaction impacts on the host

300 immune response to both natural infections and vaccines, and whether it can be manipulated

301 to enhance host immunity. Inconsistencies exist between different studies, methodologies and

302 approaches, but a growing body of evidence from humans and rodent model systems has

303 identified helminth-associated changes in gut microbiota $[43,44]$. It remains to be established

304 whether this occurs as a direct effect of the parasite itself or as a secondary effect driven by

305 the host and its immune response, or perhaps both [44]. Clearly a better understanding of co-

306 infections (in consideration also of different helminths, or of helminths and micro-

307 organisms), the mechanisms they invoke, and, importantly, their impact on the health and

308 productivity of livestock is required [45,46]. A systems biology approach, drawing insights

309 from diverse host environments (e.g. including livestock and wildlife systems), pathogen

310 combinations and stages of infection [41,44,47-49] offers promise to advance our knowledge

311 and identify potential alternative strategies for parasite control. A truly holistic view would

312 also include the impact that helminths and their control may have on other diseases and their

313 detection, including zoonoses [50].

314

315 Section V: Host resistance, resilience and selective breeding 
316 28. Have 60 years of intense anthelmintic use changed the relative susceptibility of livestock

317 to parasites? In other words, are animals less robust than they used to be as a result of

318 protection from the effects of parasites by drugs, thereby causing selection of higher-

319 producing but more parasite-susceptible animals?

32029 . How can resilience and resistance of ruminants to helminths be measured and

321 distinguished?

322 30. Is resistance, tolerance or resilience the best breeding objective to produce livestock that require less anthelmintic treatment? Under what circumstances should breeders aim for each?

324 31. Breeding for resilience (high production potential in spite of elevated faecal worm egg 325 counts) could result in significantly increased pasture contamination over many years. What

326 will the impact of higher challenges be on resilient individuals? Will the resilience break 327 down above a certain threshold?

328 32. Can targeted selective treatment, e.g. using FAMACHA, be used to select for parasite 329 resilience, especially among low-input traditional breeds?

330 33. In non-selective breeding systems, does targeted selective anthelmintic treatment support 331 weak animals and lead to loss of resilience at herd or flock level?

332 34. What are the life-time trade-offs between immunity to helminths (resistance) and impacts on growth and production (resilience) in different livestock systems?

334 35. Which are the main differences between cattle, sheep and goats in terms of resistance or 335 resilience to helminth infection?

336 36. Which genotypes of livestock hold natural resistance to helminths, and how can they be exploited in modern production systems?

338 37. Why are some animals more prone to heavy parasite burdens than others?

340 Selecting optimal host phenotypes is not straightforward 
341 Variation in susceptibility to parasites is multifactorial. Differences clearly exist between host

342 species, and these differences seem to derive from the evolutionary forces in play with regard

343 to grazing behaviors and the climate and environment where different hosts evolved.

344 However, even within host species, genetics, faecal avoidance behaviour and immunological

345 differences exist [51,52]. Moreover, the timing of measurement is important in distinguishing

346 between resistant and resilient animals as, should immunity develop, animals may thereafter

347 display a mixture of both resistance and resilience. Resistance is undoubtedly favourable

348 when faced with a fecund or highly pathogenic parasite, such as $H$. contortus [53]. In

349 contrast, resilience is associated with larger body weights and greater growth in the face of

350 helminth challenge, and can be reliably assessed based on the number of treatments required

351 using a targeted selective treatment regime [54,55]. Resilience, when it involves greater

352 tolerance of infection, generally results in greater pasture contamination, but resilient animals

353 also by definition have a greater threshold of parasite challenge before incurring loss of

354 productivity [52]. Whether the long-term epidemiological benefits of resistance outweigh the

355 missed growth opportunities remains to be determined, although the risk of pasture

356 contamination becoming too great if resilience is selected will depend on the environment

357 and grazing management, both of which influence transmission within and between seasons.

358 There are undoubtedly physiological costs to resistance and the interplay of resistance vs.

359 resilience (or tolerance) may differ between different parasite species depending on their

360 pathogenicity. These distinctions are important because hosts that are best at controlling

361 parasite burdens are not necessarily the healthiest, but can have a positive impact on the herd

362 infection levels by decreasing pasture contamination. Ultimately, resistance and

363 resilience/tolerance will have different effects not only on the epidemiology of infectious

364 diseases, but also on host-parasite coevolution [56]. The pursuit of improved host responses

365 to parasitism through selective breeding therefore requires optimization across multiple 
366 dimensions, including characteristics of the main parasites of concern now and in future,

367 production aims and farm management system, and should guard against unintended

368 consequences for co-infections.

369

370 Section VI: Development and detection of anthelmintic resistance

37138. What is the relative importance of management versus environmental factors in

372 determining the development of anthelmintic resistance in livestock?

373 39. How does animal movement affect the spread of helminth infections and anthelmintic

374 resistance?

375 40. What changes in genes other than those encoding for the immediate drug target, such as

376 transporters and drug metabolism, are involved in anthelmintic resistance?

377 41. What do we understand about the fitness costs of anthelmintic resistance and how can

378 they be measured?

379 42. Has selection for drug resistance changed the pathogenicity of parasites?

380 43. Is there a link between the size of the refugia needed to slow or prevent anthelmintic

381 resistance and the molecule and formulation used (e.g. persistent versus non-persistent)?

382 44. Can combination anthelmintic formulations be designed that are more effective and that

383 limit resistance development?

384 45. Do differences in life history traits and reproductive strategy affect the risk for

385 development of anthelmintic resistance?

386 46. What is the effect of long-lasting drug formulations such as moxidectin injections or

387 benzimidazole boluses on the development of anthelmintic resistance in sheep, goats and

388 cattle?

389 47. Is treatment of ectoparasites with macrocyclic lactone drugs an important driver of

390 anthelmintic resistance in sheep and goats? 
48. Are in-vitro/genetic/laboratory methods for detection of anthelmintic resistance desirable,

392

393

394

395

396

397

398

399

400

401

402

403

404

405

406

407

408

409

410

411

412

413

414

415 reachable and applicable for all anthelmintic drug groups?

49. How can we best improve monitoring of the efficacy of current control methods (e.g. through diagnostics, resistance testing and surveillance)?

50. How useful are composite faecal egg counts to detect anthelmintic resistance?

51. What is the true status of anthelmintic resistance in less-studied livestock systems, e.g. ascarids in pigs and poultry?

52. Is there compelling genetic evidence for reversion to drug susceptibility under any circumstances?

53. How can the prevalence of anthelmintic resistance be practically measured in a way that minimises bias?

\section{Mechanisms and processes in resistance}

The evolution of AR in parasitic helminths is considered to be driven by a range of parasite intrinsic and extrinsic factors [57]. To the former belong drug- and species-specific susceptibility, effective parasite population size and genetic variability. External factors include treatment frequency and intensity, and the size of the refugia, which strongly depend on local management and environmental determinants. How these factors interact and influence the development of a phenotypically resistant worm population is currently largely unclear. Also the molecular mechanisms of AR are not well established for most combinations of helminth species and drug groups. Nevertheless, in the case of the benzimidazoles, a well-developed understanding of the resistance mechanism has enabled molecular tools to be established for AR detection, which can be used to elucidate patterns of spread of resistance on a broad scale for ruminants [58]. The situation in pigs and poultry, however, is barely known [59]. 
417 Towards better diagnosis of anthelmintic resistance

418 There is a great need to extend our knowledge on the driving forces of AR development, to

419 establish field applicable and meaningful resistance detection tools, and hence to provide

420 more up-to-date and reliable information on the occurrence of AR. In an era of revolution of

421 technology in the diagnostic industries, improvement of the "old-fashioned" faecal egg count

422 reduction test (FECRT), for example through use of pooled faecal samples [60-62], or

423 eventually automation, has great potential to allow more rapid, labour-efficient and remote

424 assessment of AR. This remains a worthwhile aim because definitive molecular tests remain

425 elusive for most drug groups and helminth species. Better tests would enable AR to be

426 distinguished from other causes of poor efficacy, including through the administration of sub-

427 standard generic compounds [63]. Links between AR in livestock and humans, through

428 zoonotic transmission of resistant parasites such as Ascaris spp., and in terms of potential for

429 shared understanding of mechanisms and approaches to limit AR, remain underexplored.

430

431 Section VII: Practical management of anthelmintic resistance

432 When to intervene against resistance

433 54. What is the usefulness of anthelmintics working at decreased (e.g. $50 \%$ or $80 \%$ ) efficacy?

434 55. When should drug combinations be used to combat anthelmintic resistance, and when

435 not?

436

437 Optimal usage of anthelmintic drugs in the face of AR should be tailor-made and consider

438 parasite species, host species, farm management and climatic factors [2,3]. Deciding how to

439 extend the lifetime of drugs, either before or after some resistance is evident [64,65], requires

440 consideration of actual levels of AR and how fast AR spreads given selection pressures 
441 imposed by factors such as drug type and number of treatments, whether treatments are 442 targeted or not, and the presence of refugia $[66,67]$.

443

444 Refugia in principle and practice

445 56. What empirical evidence is there that refugia slow down the development of drug 446 resistance?

447 57. What proportion of a helminth population must be left in refugia in order to slow the 448 development of anthelmintic resistance?

449 58. How does the level of refugia influence the detection and spread of resistant phenotypes 450 in different hosts, different parasites and different treatment systems?

451 59. Is there a role for refugia in control of liver fluke?

452 60. If refugia are not appropriate for all parasite species that display drug resistance, what 453 realistic alternatives exist for those situations?

454 61. Can anthelmintic resistance be practically reversed, e.g. through targeted selective 455 treatment, good grazing management, or reseeding (community replacement or dilution) 456 approaches?

458 The concept of refugia is widely accepted, but is still surrounded by several assumptions and 459 approximations, and the level of refugia required may depend on prevailing (e.g. climatic) 460 circumstances [68]. Refugia as a concept has been mainly applied to GIN but its role in 461 resistance management in other helminths needs further research. Also, the extent to which 462 refugia might play a role in the reversal of AR [65], as opposed to just slowing its 463 development [69] is currently far from clear, as is the practical usefulness of community 464 replacement strategies for re-gaining anthelmintic susceptibility on farms [70]. 
What to do about known resistance status?

467 62. What is the value of faecal egg count monitoring as a decision tool for anthelmintic

468

469

470

471

472

473

474

475

476

477

478

479

480

481

482

483

484

485

486

487

488

489

490 treatments?

63. We are on the cusp of having molecular markers for drug resistance, e.g. for macrocyclic lactones in Haemonchus contortus and triclabendazole in liver fluke. How should we best apply them?

It has become common practice to apply blanket, whole-herd treatments without prior knowledge about infection levels or drug efficacy. To optimize drug usage, such prior knowledge appears to be requisite, and more science is required to create and evaluate new and more practical ways to measure levels of infection and AR.

\section{Targeting treatments against helminths}

64. Is targeted selective treatment sustainable in the long term, or will it decrease parasite overdispersion and hence ability to identify heavily infected individuals?

65. What are the most useful decision parameters in targeting anthelmintic treatments?

66. Is targeted selective treatment a feasible approach with which to control helminths with a very high biotic potential, e.g. the ascarids?

Animals within populations show different levels of susceptibility to infection both in terms of resilience and resistance, and parasites are typically over-dispersed within host groups. This opens up the path to employ targeted selective treatments of individual hosts, and in the process create and maintain refugia $[6,69]$. Treatment decision parameters need to be explored more fully; their applicability may depend on parasite species as well as host production system and much more empirical work is needed for optimisation. 
67. Can we automate interpretation of data collected during targeted selective treatment, for

494 farmer decision support and also training?

495 68. How do we apply existing knowledge of the risk factors for anthelmintic resistance on

496 farms to effectively slow its development?

497 69. What are the characteristics of an optimal quarantine drench as a way of reducing the risk

498 of importing resistance with bought in animals?

499 70. How do we implement better dosing procedures of anthelmintics in cattle in order to

500 ensure therapeutic drug levels (pour-on vs. injection/oral)?

501 71. What practical steps should be taken on a farm when resistance to all known anthelmintic drug classes develops?

504 Finally, although managing resistance through more effective targeting of treatment is an 505 intuitive approach that is becoming established best practice [6], challenges remain in terms 506 of fundamental understanding of the biological processes involved in AR. Furthermore, how 507 existing knowledge should best be integrated and structured for on-farm application, and communicated effectively through farmer and expert advisory groups (e.g. www.cattleparasites.org.uk; www.scops.org.uk; www.wormboss.com.au), itself needs a more

510 solid evidence base [9]. Effective uptake of alternative helminth management approaches

511 could not only delay AR, but also afford farmers more options if and when AR becomes

512 fixed, for example following efforts to dilute resistant alleles by introducing susceptible 513 worms [70].

\section{Section VIII: Vaccines and immunology}


516 72. Can the natural immune response to helminths be enhanced by applying a biological

517 treatment (e.g. specific cytokine or cytokine inhibitor) and thereby control infections?

518 73. Do worms have a microbiome? Can it be exploited as a vaccine or treatment target?

519 74. How can vaccines against helminth infections in ruminants be integrated in control

520 programmes?

52175 . In what ways do helminths resist or escape from the host immune system?

52276 . How well do anti-helminth vaccines have to work to be useful?

523 77. To what extent is the immunomodulation by helminth parasites detrimental to the

524 animal's health when co-infections co-occur?

525 78. What mechanisms are involved in protective immunity against helminths?

526 79. What is the potential for a multivalent vaccine to control multiple species?

52780 . How are optimal helminth vaccination schedules influenced by infection pressure and can

528 this be incorporated into decision making?

529 81. How fast do parasites adapt to increased immune selection pressures (for instance due to $530 \quad$ vaccines)?

531

More insight needed into natural immune responses

533 Helminths typically induce a T-helper 2 type immune response, but the effector mechanisms

534 have not yet been elucidated and it is not always clear whether this immune response is host

535 protective or to the advantage of the parasite, which is acknowledged as a major knowledge

536 gap [8]. Incomplete knowledge about protective immune responses against helminths

537 hampers vaccine development. Insight into the immune mechanisms would allow informed

538 decisions about adjuvants and antigen delivery [71] and could lead to alternative immune

539 therapies, e.g. cytokines or cytokine inhibitors, which has shown potential in porcine

540 neurocysticercosis [72]. 
Integrating vaccines into control programmes

543 To be useful alternatives to anthelmintics, vaccines should protect against multiple helminth

544 species [71]. At present, there is only one vaccine for gastrointestinal nematodes available;

545 targeting Haemonchus contortus (http://barbervax.com.au/), and other experimental vaccines

546 are also limited to single species and there is no evidence for cross-protection, e.g. between

547 Cooperia and Ostertagia in cattle [73]. 'Multivalent' vaccines could also include those

548 containing multiple antigens of a single parasite species, to avoid or slow down adaptation of

549 the parasites to the vaccine, e.g. an experimental Teladorsagia vaccine in sheep that

550 comprises multiple recombinant proteins [71]. To protect young animals until natural

551 immunity has developed, vaccines should lower pasture infection levels by reducing worm

552 egg output in vaccinated animals for a useful period [74]. The level and duration of protection

553 needed will be different for different parasites and in different epidemiological settings, e.g.

554 on pastures with high or low infection pressure, and may differ with changing climate or farm

555 management.

556 Vaccination, even if only partially effective could become an important component of

557 integrated worm control programmes, including pasture management and anthelmintic

558 treatment [1]. The huge number of possible scenarios could be investigated using helminth

559 transmission models [75-79]. After field validation, these models could ultimately lead to

560 decision support software for integrated worm control [9]. The sustainability of vaccines, like

561 anthelmintics, will depend on parasite evolution, and the ability of helminths to develop

562 resistance to vaccine-induced host responses remains an open question.

564 Section IX: Alternative approaches to helminth management

565 Plant-based control 
82. Many studies have shown a maximum efficacy of bioactive plant compounds around 60-

$56770 \%$ reduction in gastrointestinal nematode burden: how can efficacy be driven higher? Is it needed?

83. Can different bioactive plants be combined to increase effects on gastrointestinal nematodes?

84. Can plants be cultivated for grazing that have maximum nutritive value and the potential to lower helminth burden?

85. How does processing and conservation of bioactive forages affect their efficacy?

86. What are the interactions between bioactive forages and synthetic anthelmintic drugs, in vitro and in vivo?

87. What are the mechanisms of action of bioactive plant compounds and metabolites in relation to parasite establishment and adult worm viability and fecundity?

88. What is the efficacy of plant based anthelmintics against drug resistant helminths?

580 With the increasing emergence of AR in helminths of livestock, alternative options are in

581 demand, especially for the integrated control of GINs. Plants and their Secondary Metabolites

582 (PSM) appear to be a promising option. Different PSM (e.g. tannins) have shown antiparasitic effects when used as nutraceuticals [80] or in phytotherapy [81]. Two hypotheses have been invoked to explain the anthelmintic properties of PSM [82]:

585 pharmacological-like effects through disturbance of the parasite life-cycle [83], or indirect 586 effects on the host immune response [84]. In both cases, more studies are needed to identify the mechanisms of action of PSM and their effect on helminth populations, including those with high levels of AR, as well as the potential role of PSM in managing helminths other than GINs. Feeding 'bioactive forages' can also improve nutrition and performance, and reduce

590 GHG emissions, quite apart from any impacts on helminths. 
591 The interactions between different PSM and between PSM and anthelmintics remain largely unexplored and contrasting results have been described [85]. The development of refined methods to assess the anthelmintic potential of plant compounds are needed. Some

594 practicalities around use of PSM on farms also need to be addressed, such as regulation of mode of distribution, level of inclusion in feed, and potential residues in animal products.

\section{Other alternative control methods}

89. What are the main obstacles (not only technical) to the development of new technologies to control helminths of livestock?

90. Can we target helminth stages outside the host to achieve control, e.g. killing stages on pasture or manipulating intermediate host biology?

91. Are there basic processes in egg hatching or larval development that can be manipulated to aid control?

605 The objective of integrated parasite management is to limit the level of parasitism below acceptable limits while delaying the emergence of drug resistance. This aim has motivated the search for and refined use of PSM as well as other alternatives to commercial chemical anthelmintics, including vaccines, host resistance and grazing management [86]. Good pasture management is one of the major means to limit the intake of infective larvae by animals, e.g. by use of parasite-free fields, pasture rotations, and alternation of grazing

611 animals, taking into account the seasonal dynamics of helminth transmission. Manipulation

612 of environmental conditions that play a role in the development of intermediate stages may

613 also be a form of alternative control. For example, grazing away from wet pasture, where

614 feasible, markedly lowers the risk of $F$. hepatica infection, due to lower exposure to infection 615 near intermediate snail host habitats [87]. Free-living stages of GIN may also be targeted 
616 directly, for instance through application of urea or other nitrogen-based fertilisers to pasture

617 [88,89]. Certain bioactive forages, e.g. chicory, are also thought to hamper the development

618 of free-living stages, either by reducing the fitness of eggs excreted from hosts grazing on the

619 forage, or because the physico-chemical properties of the forage reduce larval availability on

620 herbage [90]. Biological control based on nematode trapping fungi (Duddingtonia flagrans,

621 Arthrobotrys musiformis) or entomopathogenic bacteria can also reduce the number of free

622 living stages on pasture and the level of host infections; results from mechanical stressors

623 such as a diatomaceous earth are less promising [91,92]. Refined understanding of the

624 mechanisms of action of these non-chemotherapeutic alternative control methods and how

625 they might be applied to manage helminth populations on farms provide potentially fruitful

626 avenues for further research.

627

628 Section X: Stakeholder engagement

629 New decision support tools for helminth control

630 92. How can different novel control methods for helminths be integrated effectively and in a

631 way that is simple enough for farmers to implement?

632 93. Can helminth control decision support tools be integrated effectively in farm or pasture

633 management software?

634 94. How can we transfer automated technology to farmers, especially those that are resource635 poor?

636 95. Is research in veterinary helminth infections reaching livestock farmers in developing 637 countries and, if so, what is the impact?

638

639 Veterinary parasitologists working with livestock might consider extending their efforts from

640 task-oriented research targeting the development and refinement of helminth control 
641 strategies, and advance towards advice-oriented health management practices. To achieve this

642 would involve answering some key research questions around development of decision

643 support tools that can integrate different worm control strategies into whole-farm

644 management [9], taking into account also the regulatory frameworks and economic

645 environments in which farmers operate. Researchers are now looking further down this road

646 and questioning how their strategies will fit best into the whole farm environment and how

647 decision tools can be integrated, for example in farm management practices and decision

648 support systems. Even though there is considerable knowledge on available complementary

649 strategies, substantial deficits remain around knowledge exchange and transfer, and the

650 research community is becoming increasingly aware that better promotion of such strategies

651 to the farmers is crucial for their success [93].

652

653 Understanding farmer behaviour to support effective knowledge exchange

654 96. What factors drive anthelmintic treatment decisions by farmers?

65597. How can the importance of a strategic approach to helminth treatment be more effectively

656 promoted among producers, especially when drug resistance is not yet an issue?

657 98. What can we learn from social sciences to transfer knowledge on helminth control to

658 farmers?

659 99. How does the attitude of farmers with respect to accepting and implementing parasite

660 control measures differ between countries and cultures?

661 100. How will consumers influence livestock production practices, in terms of anthelmintic 662 use?

663

664 In order to develop control methods that are effectively applied, it is necessary to obtain 665 insights into factors that drive farmers' decisions about worm control and use those insights 
to develop communication strategies to promote sustainable worm control practices [94].

667 Major reasons why suggested solutions often do not fit with farmers' views are that they are

668 highly complex (involving language and cultural barriers) and not cost-efficient (too

669 expensive), encompass conflicting interests (e.g. intensive versus extensive farming systems)

670 and priorities, and may require contradictory management interventions at farm level.

671 Consequently, educating and motivating farmers and adopting a multi-actor approach are key

672 issues. Stronger empirical evidence for the effectiveness of integrated parasite control

673 strategies and their compatibility with performance targets is key to adoption $[94,95]$.

674 Researchers must understand the fundamental and instrumental relationships between

675 individual farmers' values, behaviour and perception of risk, to stimulate and qualify the

676 farmer's decision-making in a way that will increase the farmer's satisfaction and subjective

677 well-being, and not only narrow metrics around performance or financial return $[26,96]$.

678 Factors that influence farmers' behaviour are not limited to technical or practical issues such

679 as ease of use or price, but also include less 'tangible' factors such as the opinion of others or

680 habits [97-99]. Barriers and incentives for sustainable worm control that were identified in

681 such quantitative and qualitative studies may vary between farmer types (e.g. sheep farmers

682 vs. dairy cattle farmers) or between countries. Moreover, before these factors can be

683 translated into communication strategies, they should first be validated in communication

684 experiments [100]. In the literature on changing animal health behaviour, the majority

685 comprises studies that investigate the factors that influence behaviour intention, which at best

686 suggests which social intervention could be developed to change this intended behaviour, but

687 rarely assess whether such intervention could work [101]. Finally, human behaviour (and thus

688 also farmer behaviour) is also strongly influenced by unconscious processes, such as

689 intuition, which has not yet been studied in the context of sustainable parasite control [102]. 
690 As a community, veterinary parasitologists need to adopt a trans-disciplinary approach,

691 together with epidemiologists, social scientists, economists and others (including livestock

692 scientists, grassland management experts, conservationists, processors, retailers and farmers

693 themselves), which will result in a better understanding of farmer behaviour and motivation

694 with respect to drug treatments and parasite control.

695

696 Concluding remarks

697 The questions listed above were the result of an attempt to elicit research priorities from a

698 wider constituency than in more usual review formats, which are typically led by a small

699 number of established experts. It was anticipated that this would yield a wider-ranging set of

700 potential research topics and directions, less constrained by forces that shape disciplinary

701 academic consensus. In the event, the topics and questions are broadly similar to those raised

702 in recent expert reviews [1,4,6-8,103], and reflect a high level of current concern over the

703 biology of AR, how to measure and manage it, and the quest for alternative options for the

704 control of helminths on farms. This is perhaps not surprising given that improved helminth

705 management is a key goal of most researchers in the discipline, whether they lean toward

706 fundamental or applied research, and that AR is the main threat to existing control strategies.

707 Control of helminth infections in mainstream farming systems with fewer chemical inputs is

708 a topical challenge and one that will require new research, technologies, and perhaps

709 economic goals [1].

710 Questions around helminth epidemiology, management of AR, and alternative control

711 approaches including refugia, were frequently repeated in the original list (see supplemental

712 material), for example being posed more than once for different parasite or host taxa. To

713 achieve feasible smaller research projects as envisaged at the start of this exercise, many of

714 the questions could be broken back down again to specific taxa, both to produce system- 
715 specific knowledge and applied solutions, and to explore the generality of conclusions from more studied contexts. Challenges in tropical or less developed countries yielded few specific

717 questions, as did those related to pig and poultry production. Participation was strongly

718 skewed towards European countries, in spite of efforts to be inclusive, possibly as a result of

719 the European roots of LiHRA, under whose auspices the exercise was conducted (Box 1).

720 Nevertheless, questions submitted from outside Europe focused on similar areas, and almost

721 all of the final questions are relevant across wide geographic areas and often globally. The voting round (Box 2) might also have distorted results and led to the loss of original but less popular ideas from the final list, though such a step was necessary to limit numbers of questions and exclude some to which answers are already well-known. The full list is included as supplemental material to this article.

While not definitive, the final list of 100 questions serves to indicate current concerns among the livestock helminth research community, and highlights several areas in which existing understanding is poor while fresh advances now appear possible. The questions might serve to encourage or inspire work in those areas. For example, early career researchers might peruse the list to identify topics on which short or starter projects might have disproportionately high impact on the state of knowledge. It would be instructive to repeat this exercise in future, to determine how many of the questions have been answered, and whether the state of knowledge, the enabling technologies, or the problems of the day have moved sufficiently to generate different gaps and priorities. In the meantime, as a community, there is clearly work to be done to explore interesting questions whose answers are highly relevant to the ability of humankind to feed itself in the future while respecting the global environment and the health and welfare of the animals that sustain us. 
740 We thank the officers and members of the Livestock Helminth Research Alliance (LiHRA)

741 for encouraging this initiative and giving space to it in their annual meetings, to the World

742 Association for the Advancement of Veterinary Parasitology for permitting elicitation of

743 questions as part of their 26th biennial conference in Kuala Lumpur, Malaysia, and to the

744 editors of Trends in Parasitology for commissioning this article. The authors credit the EU for

745 funding leading to this work through FP7 STREP GLOWORM. We gratefully acknowledge

746 Hassan Azaizeh, Sarah Beynon, Jacques Cabaret, Gerald Coles, Tina Alstrup Hansen, Alison

747 Howell, Hamadi Karembe, Alvaro Martinez-Moreno, Francisco A. Rojo, Guillaume Sallé,

748 Jože Starič, Eurion Thomas, and numerous anonymous participants for contributing

749 generously to the exercise with questions and votes. This article is based in part upon work

750 from COST Action COMBAR CA16230, supported by COST (European Cooperation in

751 Science and Technology). MMV is funded by the Spanish "Ramón y Cajal" Programme,

752 Ministry of Economy and Competitiveness (RYC-2015-18368), and ERM and HRV by UK

753 BBSRC grant BB/M003949/1.

754

\section{References}

756 [1] Vercruysse J. et al. (2018) Control of helminth ruminant infections by 2030. Parasitology

757 doi: 10.1017/S003118201700227X

758 [2] Kaplan, R.M. and Vidyashankar, A.N. (2012) An inconvenient truth: global worming and

759 anthelmintic resistance. Vet. Parasitol. 186, 70-78

760 [3] Rose, H. et al. (2015) Widespread anthelmintic resistance in European farmed ruminants:

761 a systematic review. Vet. Rec. 176, 546

762 [4] Sangster, N.C. et al. (2018) Ten events that defined anthelmintic resistance research.

763 Trends Parasitol. DOI: https://doi.org/10.1016/j.pt.2018.05.001 
764 [5] Van Dijk, J. et al. (2008) Climate change and infectious disease: helminthological

765 challenges to farmed ruminants in temperate regions. Animal 4, 377-392

766 [6] Charlier, J., et al. (2014). Practices to optimise gastrointestinal nematode control on

767 sheep, goat and cattle farms in Europe using targeted (selective) treatments. Vet. Rec. 175,

$768 \quad 250$

769 [7] Beesley, N.J. et al. (2018). Fasciola and fasciolosis in ruminants in Europe: Identifying 770 research needs. Transbound. Emerg. Dis. 256, 199-216

771 [8] Charlier, J. et. al. (2018) Mind the gaps in research on gastrointestinal nematodes of

772 veterinary importance. Transbound. Emerg. Dis. 65, 217-234

773 [9] Van Wyk, J.A. et al. (2011) Blueprint for an automated specific decision support system

774 for countering anthelmintic resistance in Haemonchus spp. at farm level. Vet. Parasitol. 177, $775 \quad 212-223$

776 [10] Sutherland, W.J. et al. (2013) Identification of 100 fundamental ecological questions. J.

777 Ecol. 101, 58-67

778 [11] Gibbs, H.C. (1986) Hypobiosis in parasitic nematodes - an update. Adv. Parasitol. 25, $779 \quad 129-174$

780 [12] Langrova, I. et al. (2008). Arrested development of sheep strongyles: onset and

781 resumption under field conditions of Central Europe. Parasitol. Res. 103, 387-392

782 [13] Cantacessi, C. et al. (2010) Deep insights into Dictyocaulus viviparus transcriptomes

783 provides unique prospects for new drug targets and disease intervention. Biotechnol Adv. 29:

784 10.1016/j.biotechadv.2010.11.005

785 [14] Rowe, A. et.al. (2008) Haemonchus contortus infection in sheep: Parasite fecundity

786 correlates with worm size and host lymphocyte counts. Vet. Parasitol. 153, 285-293

787 [15] Walker, J.G. and Morgan, E.R. (2014) Generalists at the interface: Nematode transition

788 between wild and domestic ungulates. Int. J. Parasitol. Parasites Wildl. 3, 242-250 
[16] Armour, J. et al. (1988). Clinical nematodiriasis in calves due to Nematodirus battus infection. Vet. Rec. 123, 230-231

791

[17] Pyziel, A.M. et al. (2016) Interrelationships of Dictyocaulus spp. In wild ruminants with morphological description of Dictyocaulus cervi N. sp. (Nematoda: Trichostrongyloidea)

793 from red deer, Cervus elaphus. J. Parasitol. 103, 506-518

794 [18] Walker, J.G. et. al. (2018) Prediction and attenuation of seasonal spill over of parasites 795 between wild and domestic ungulates in an arid mixed-use system. J. Appl. Ecol. 55, 1976-

796 1986

797 [19] Chintoan-Uta, c. et al. (2014) Wild deer as potential vectors of anthelmintic-resistant abomasal nematodes between cattle and sheep farms. Proc. R. Soc. B. Biol. Sci. 281, 799 20132985

800 [20] Cable, J. et al. (2017) Global change, parasite transmission and disease control: lessons

801 from ecology. Phil. Trans. R. Soc. B 372, 20160088

802 [21] Gethings, O.J. et al. (2015) Asynchrony in host and parasite phenology may decrease 803 disease risk in livestock under climate warming: Nematodirus battus in lambs as a case study.

804 Parasitology 142, 1306-1317

805 [22] Koelle, K. et al. (2005) Pathogen adaptation to seasonal forcing and climate change.

806 Proc. R. Soc. B 272, 971-977

807 [23] Huson, K. et al. (2017) Paramphistomosis of ruminants: an emerging parasitic disease in 808 Europe. Trends Parasitol. 33, 836-844

809 [24] Escarcha, J. et al. (2018) Livestock under climate change: a systematic review of

810 impacts and adaptation. Climate. 6. Dpi: 10.3390/cli6030054

811 [25] Molento, M. et al. (2016) Pasture larval count as a supporting method for parasite 812 epidemiology, population dynamic and control in ruminants. Livest. Sci. 192, 48-54 
813 [26] Charlier J. et. al. (2015) ECONOHEALTH: placing helminth infections of livestock in

814 an economic and social context. Vet. Parasitol. 212, 62-67

815 [27] Kenyon, F. et al. (2013) Reduction in greenhouse gas emissions associated with worm

816 control in lambs. Agriculture 3, 271-284

817 [28] Houdijk, J.G.M. et al. (2011) Animal health and greenhouse gas intensity: the paradox of

818 periparturient parasitism. Int. J. Parasitol. 47, 633-641

819 [29] Williams, A.R. et al. (2015) A systems-life cycle assessment approach to modelling the

820 impact of improvements in cattle health on greenhouse gas emissions. Adv. Anim. Biosci. 6,

$821 \quad 29-31$

822 [30] Cooke, A.S. et al. (2017) Modelling the impact of targeted anthelmintic treatment of

823 cattle on dung fauna. Environ. Toxicol. Pharmacol. 55, 94-98

824 [31] Verdu, J.R. et al. (2018) Ivermectin residues disrupt dung beetle diversity, soil properties

825 and ecosystem functioning: An interdisciplinary field study. Sci. Total Environ. 618, 219-228

826 [32] Herrero, M. et al. (2015) Livestock and the environment: What have we learned in the

827 past decade? Annu. Rev. Environ. Resour. 40, 177-202

828 [33] Perry, B.D. and Randolph, T.F. (1999) Improving the assessment of the economic

829 impact of parasitic diseases and of their control in production animals, Vet. Parasitol. 84,

$830 \quad 145-168$

831 [34] Forbes, A.B., et al. (2000) Evaluation of the effects of nematode parasitism on grazing

832 behaviour, herbage intake and growth in young grazing cattle. Vet. Parasitol., 90, 111-118

833 [35] Moore, J. (2002) Parasites and the behavior of animals. Oxford Series in Ecology and

834 Evolution. ISBN: 0-19-508441-1

835 [36] Szyszka, O. et al. (2013) Do the changes in the behaviours of cattle during parasitism

836 with Ostertagia ostertagi have a potential diagnostic value? Vet. Parasitol. 193, 214-222

837 [37] Hutchings, M.R. et al. (1998) Behavioural strategies used by parasitized and non- 
838 parasitized sheep to avoid ingestion of gastro-intestinal nematodes associated with faeces.

839 Anim. Sci. 67, 97-106

840 [38] Hoste H. et al. (2010) Goat-nematode interactions: think differently. Trends Parasitol.

$841 \quad 26,376-381$

842 [39] Giacomin, P. et al. (2015) Suppression of inflammation by helminths: a role for the gut

843 microbiota? Phil. Trans. R. Soc. B. Biol. Sci. 370, 1675, 0140296

844 [40] Grenfell, B.T. (1988) Gastrointestinal nematode parasites and the stability and

845 productivity of intensive ruminant grazing systems. Phil. Trans. R. Soc. Lond. B. Biol. Sci.

$846 \quad 321,541-563$

847 [41] Garza-Cuartero, L. Et al. (2014) The worm turns: trematodes steering the course of co-

848 infections. Vet. Path. 51, 385-392

849 [42] Gause, W.C., and Maizels, R.M. (2016) Macrobiota-helminths as active participants and

850 partners of the microbiota in host intestinal homeostasis. Curr. Opin. Microbiol. 32, 14-18

851 [43] Midha, A. et al. (2017) Reciprocal interactions between nematodes and their microbial

852 environments. Front. Cell. Infect. Microbiol. 7, 1-20

853 [44] Peachey, L.E. et al. (2017) This gut ain't big enough for the both of us. Or is it?

854 Helminth-microbiota interactions in veterinary species. Trends Parasitol. 33, 619-632

855 [45] Steenhard, N.R. et al. (2009). Ascaris suum infection negatively affects the response to a

856 Mycoplasma hyopneumoniae vaccination and subsequent challenge in pigs. Vaccine, 27,

$857 \quad 5161-5169$

858 [46] Andreasen, A. et al. (2015) Immune and inflammatory responses in pigs infected with

859 Trichuris suis and Oesophagostomum dentatum. Vet. Parasitol. 207, 249-258

860 [47] Ezenwa, V.O. (2016) Helminth-microparasite co-infection in wildlife: lessons from

861 ruminants, rodents and rabbits. Parasit. Immunol. 38, 527-534 
862 [48] Peachey, L.E. et al. (2018) The relationships between faecal egg counts and gut

863 microbial composition in UK Thoroughbreds infected by cyathostomins. Int. J. Parasitol.

864 Doi: 10.1016/j.ijpara.2017.11.003

865 [49] Clark et al 2018. Strongyle infection and gut microbiota: profiling of resistant and

866 susceptible horses over a grazing season. Front. Physiol. 9, 272

867 [50] Claridge J. et al. (2012) Fasciola hepatica is associated with the failure to detect bovine

868 tuberculosis in dairy cattle. Nat. Commun. 3, 853

869 [51] Hutchings, M. et al. (2007) Genetically resistant sheep avoid parasites to a greater extent

870 than do susceptible sheep. Proc. R. Soc. Lond. B Biol. Sci. 274, 1839-1844

871 [52] Greer, A.W. et al. (2018) Immune development and performance characteristics of

872 Romney sheep selected for either resistance or resilience to gastrointestinal nematodes. Vet.

873 Parasitol. 250, 60-67

874 [53] Riley, D.G. and Van Wyk, J.A. (2009) Genetic parameters for FAMACHA (c) score and

875 related traits for host resistance/resilience and production at differing severities of worm

876 challenge in a Merino flock in South Africa. Vet. Parasitol. 164, 44-52

877 [54] Bisset S.A. et al. (1994) Genetics of resilience to nematode parasites in Romney sheep.

878 N.Z. J. Agric. Res. 37, 521-534

879 [55] Greer, A.W. et al (2013) Evaluation of lambs subjected to a targeted selective treatment

880 anthelmintic regime. Proc. N.Z. Soc. Anim. Prod. 73, 175-179

881 [56] Raberg, L. Et al. (2009) Decomposing health: tolerance and resistance to parasites in

882 animals. Philos. Trans. R. Soc. B Biol. Sci. 364, 37-49

883 [57] Wolstenholme, A.J. et al. (2004) Drug resistance in veterinary helminths. Trends

884 Parasitol. 20, 469-476 
885 [58] Knapp-Lawitzke, F. et al. (2015) Rapid selection for $\beta$-tubulin alleles in codon 200

886 conferring benzimidazole resistance in an Ostertagia ostertagi isolate on pasture. Vet.

887 Parasitol. 209, 84-92

888 [59] Tarbiat, B. et al. (2017) Evaluation of benzimidazole resistance status in Ascaridia galli.

889 Parasitology 144, 1338-1345

890 [60] Rinaldi, L. et al. (2014) Comparison of individual and pooled faecal samples in sheep for 891 the assessment of gastrointestinal strongyle infection intensity and anthelmintic drug efficacy

892 using McMaster and Mini-FLOTAC. Vet. Parasitol. 205, 216-223

893 [61] Kenyon, F. et al. (2016) Pooling sheep faecal samples for the assessment of anthelmintic

894 drug efficacy using McMaster and Mini-FLOTAC in gastrointestinal strongyle and

895 Nematodirus infection. Vet. Parasitol. 225, 53-60

896 [62] George, M. et al. (2017) Utilization of composite fecal samples for detection of

897 anthelmintic resistance in gastrointestinal nematodes of cattle. Vet. Parasitol. 240, 24-29

898 [63] Wyk, J.A. van (1997) Quality control in generic anthelmintics: Is it adequate? Vet.

899 Parasitol., 72, 157-165

900 [64] Learmount, J., et al. (2012). A computer simulation study to evaluate resistance

901 development with a derquantel-abamectin combination on UK sheep farms. Vet. Parasitol.

$902 \quad 187,244-253$

903 [65] Leathwick, D. M. et al. (2015) Evidence for reversion towards anthelmintic

904 susceptibility in Teladorsagia circumcincta in response to resistance management

905 programmes. Int. J. Parasitol. Drugs Drug Resist. 5, 9-15

906 [66] Wyk, J.A. van (2001) Refugia - overlooked as perhaps the most potent factor concerning

907 the development of anthelmintic resistance. Onderstepoort J. Vet. Res. 68, 55-67 
908 [67] Kenyon, F. et al. (2009) The role of targeted selective treatments in the development of

909 refugia-based approaches to the control of gastrointestinal nematodes of small

910 ruminants. Vet. Parasitol. 164, 3-11

911 [68] Cornelius, M.P. et al. (2016) Computer modelling of anthelmintic resistance and worm

912 control outcomes for refugia-based nematode control strategies in Merino ewes in Western

913 Australia. Vet. Parasitol. 220, 59-66

914 [69] Kenyon, F. et al. (2013) A comparative study of the effects of four treatment regimes on

915 ivermectin efficacy, body weight and pasture contamination in lambs naturally infected with

916 gastrointestinal nematodes in Scotland. Int. J. Parasitol. Drugs Drug Res. 3, 77-84

917 [70] Muchiut M. et al. (2018) Anthelmintic resistance: Management of parasite refugia for

918 Haemonchus contortus through the replacement of resistant with susceptible populations.

919 Vet. Parasitol. 254, 43-48

920 [71] Matthews J.B. et al. (2016) Progress in the development of subunit vaccines for

921 gastrointestinal nematodes of ruminants: tools and technologies. Parasite Immunol. 38, 744-

$922 \quad 753$

923 [72] Mahanty, S. et.al. (2017) TNF- $\alpha$ blockade suppresses pericystic inflammation following 924 anthelmintic treatment in porcine neurocysticercosis. PLoS Negl. Trop. Dis. 11, e0006059

925 [73] Vlaminck J. et al. (2015) Vaccination of calves against Cooperia oncophora with a

926 double-domain activation-associated secreted protein reduces parasite egg output and pasture

927 contamination. Int. J. Parasitol. 45, 209-213

928 [74] Claerebout, E. et al. (2003) Current research and future prospects in the development of 929 vaccines against gastrointestinal nematodes in cattle. Exp. Rev. Vaccines 2, 147-157

930 [75] Rose, H. et al. (2015) GLOWORM-FL: A simulation model of the effects of climate and

931 climate change on the free-living stages of gastro-intestinal nematode parasites of ruminants.

932 Ecol. Model. 297, 232-245 
933 [76] Turner, J. et al. (2016) A model to assess the efficacy of vaccines for control of liver

934 fluke infection. Sci. Rep. 6, 23345

935 [77] Sauermann, C.W. and Leathwick, D.M. (2018) A climate-driven model for the dynamics

936 of the free-living stages of Cooperia oncophora. Vet. Parasitol. 255, 83-90

937 [78] Beltrame, L. et al. (accepted) A mechanistic hydro-epidemiological model of liver fluke

938 risk. J. R. Soc. Interface

939 [79] Verschave, S.H. et al. (2016) Cattle and nematodes under global change: transmission

940 models as an ally. Trends Parasitol. 32, 724-738

941 [80] Hoste, H. et. al. (2015) Tannin containing legumes as a model for nutraceuticals against

942 digestive parasites in livestock. Vet. Parasitol. 212, 5-17

943 [81] Ropiak, H.M. et al. (2016) Condensed tannins in extracts from European medicinal

944 plants and herbal products. J. Pharma. Biomed. Anal. 120, 225-231

945 [82] Hoste, H. et al. (2012) Direct and indirect effects of bioactive legume forages against

946 parasitic infections: experiences with tropical and temperate forages. Vet. Parasitol. 186, 18-

$947 \quad 27$

948 [83] Williams, A.R. et al. (2014) Direct anthelmintic effects of condensed tannins from

949 diverse plant sources against Ascaris suum PLoS One 9, e97053

950 [84] Williams, A.R. et al. (2017) Dietary cinnamaldehyde enhances acquisition of specific

951 antibodies following helminth infection in pigs. Vet. Immunol. Immunopathol. 189, 43-52

952 [85] Gaudin, E. et al. (2016) Efficacy of sainfoin (Onobrychis viciifolia) pellet against multi

953 resistant $H$. contortus and interaction with oral ivermectin: implication for on-farm control.

954 Vet. Parasitol. 227, 122-129

955 [86] Šimpraga, M. et al. (2015) Alternative approaches for the control of gastrointestinal

956 nematodes in sheep farming: a review. Berl. Munch. Tierarztl. Wochenschr. 128, 257-7070 
[87] Takeuchi-Storm et al. (2017) Farm-level risk factors for Fasciola hepatica infection in

958 Danish dairy cattle as evaluated by two diagnostic methods. Parasit. Vectors, v10, 555

959

[88] Howell et al. (1999) Control of gastrointestinal parasite larvae of ruminant using

960 nitrogen fertilizer, limestone and sodium hypochlorite solutions. Small Rumin. Res. 32, 197-

961204

962 [89] Cairns et al. (2017) Potential anthelmintic properties of urea. Proc. New Zealand Soc.

963 Anim. Prod. 77, 110-113

964 [90] Marley et al. (2003) The effect of dietary forage on the development and survival of

965 helminth parasites in ovine faeces. Vet. Parasitol., 93-107

966 [91] Ahmed, M.A. et al. (2013) Studies on the ability of two isolates of Bacillus

967 thuringiensis, an isolate of Clonostachys rosea f. rosea and a diatomaceous earth product to 968 control gastrointestinal nematodes of sheep. Biocontrol Sci. Technol. 23, 1067-1082

969 [92] Da Silveira, W.F. et al. (2017) Nematophagous fungi combinations reduce free-living

970 stages of sheep gastrointestinal nematodes in the field. J. Invert. Pathol. 150, 1-5

971 [93] Charlier et al. (2016). Decision making on helminths in cattle: diagnostics, economics

972 and human behaviour. Ir. Vet. J. 69:14

973 [94] Woodgate, R.G. and Love, S. (2012) WormKill to WormBoss: can we sell sustainable 974 sheep worm control? Vet. Parasitol. 186, 51-57

975 [95] Van Wyk, J.A. (2008) Production trials involving use of the FAMACHA (c) system for 976 haemonchosis in sheep: preliminary results. Onderstepoort J. Vet. Res. 75, 331-345

977 [96] Learmount, J. et al. (2018) Resistance delaying strategies on UK sheep farms: A cost 978 benefit analysis. Vet. Parasitol. 254, 64-71

979 [97] Van de Velde et al. (2015) Diagnosis before treatment: Identifying dairy farmers' 980 determinants for the adoption of sustainable practices in gastrointestinal nematode control.

981 Vet. Parasitol. 212, 308-317 
982 [98] Jack et al. (2017) A quantitative analysis of attitudes and behaviours concerning

983 sustainable parasite control practices from Scottish sheep farmers. Prev. Vet. Med. 139, 134-

$984 \quad 145$

985 [99] Van de Velde, F. et al. (2018) Beliefs, intentions, and beyond: A qualitative study on the 986 adoption of sustainable gastrointestinal nematode control practices in Flanders' dairy

987 industry. Prev. Vet. Med. 153, 15-23

988 [100] Van de Velde et al. (2018) Changing farmers' behavior intention with a hint of wit: The 989 moderating influence of humor on message sidedness. Environ. Psychol. 56, 97-103

990 [101] Ritter, C. et al. (2017) Determinants of farmers' adoption of management-based

991 strategies for infectious disease prevention and control. J. Dairy Sci. 100, 3329-3347

992 [102] Kristensen, E. and Jakobsen, E.B. (2011) Challenging the myth of the irrational dairy 993 farmer; understanding decision-making related to herd health. N. Z. Vet. J. 59, 1-7

994 [103] Charlier J. et al. (2014) Recent advances in the diagnosis, impact on production and 995 prediction of Fasciola hepatica in cattle. Parasitology 141, 326-335

996 [104] O’Brien, D. et al. (2017) DISCONTOOLS: a database to identify research gaps on 997 vaccines, pharmaceuticals and diagnostics for the control of infectious diseases of animals. 998 BMC Vet. Res. 13, 1

999 
1001 Europe.

1002 Deciding where public and private research spending will have the greatest impact is a 1003 complex process involving multiple interests. Often, ad hoc expert groups are created to

1004 provide decision makers with advice over specific topics. In addition, over the last decade 1005 several initiatives have emerged at European and global levels to foster international 1006 discussions and apply a structured approach to the identification of research gaps and

1007 priorities in the animal health domain, including livestock helminthology in Europe.

1008 DISCONTOOLS (www.discontools.eu) is a publicly funded, open-access database to assist

1009 public and private funders of animal health research and researchers in identifying research 1010 gaps and planning future research [104]. The database contains research gaps as well as a gap

1011 scoring and prioritization model for more than 50 infectious diseases of animals. The

1012 information is provided by disease-specific expert groups and updated on a 5-year cycle.

1013 The DISCONTOOLS database acts as a key resource for the STAR-IDAZ International

1014 Research Consortium on animal health (www.star-idaz.net), comprising research funders and

1015 programme owners from Europe, Asia, Australasia, the Americas, Africa and the Middle

1016 East, as well as international organisations, and includes representation from veterinary

1017 pharmaceutical companies. Members coordinate their research programmes to address agreed

1018 research needs, share results, and together seek new and improved animal health strategies

1019 for at least 30 priority diseases, infections or issues. These include candidate vaccines,

1020 diagnostics, therapeutics and other animal health products, procedures and key scientific

1021 information and tools to support risk analysis and disease control. STAR-IDAZ develops

1022 road maps on how to achieve these new animal health strategies.

1023 The Animal Task Force (ATF) (www.animaltaskforce.eu) is a European public-private

1024 platform that fosters knowledge development and innovation for a sustainable and 
1025 competitive livestock sector in Europe. It represents key stakeholders from industry, farmers

1026 and research from across Europe. It is a knowledge-based lobby organisation working at the

1027 forefront of livestock related issues in Europe, including but not limited to animal health

1028 issues. The ATF unites members from every aspect of the livestock value chain (from feeding

1029 and breeding to production and processing), enabling an integrated approach to contribute to

1030 the environmental and societal challenges of livestock systems.

1031 The Livestock Helminth Research Alliance (LiHRA) (www.lihra.eu) is a consortium of

1032 researchers that aims to develop sustainable effective helminth control strategies and promote

1033 their implementation by the livestock industry. LiHRA grew out of EU-funded research

1034 projects addressing challenges in the control of gastrointestinal nematodes (FP6 PARASOL)

1035 and liver fluke (FP6 DELIVER) in ruminants under global change (FP7 GLOWORM), and

1036 related projects investigating alternative control approaches (Marie-Curie Initial Training

1037 Networks NematodeSystemHealth, Healthy Hay and Legume Plus, www.legumeplus.eu).

1038 LiHRA meets annually to review current challenges, recent results and opportunities for

1039 collaborative research. Discussions within LiHRA gave rise to the current article, and also

1040 underpinned the EU-funded networking COST Action COMBAR. 
1044 The questions presented in this article were elicited in a way intended to be inclusive and to 1045 encourage participation from a diverse range of researchers, regardless of career stage, gender 1046 or geographical location. Initially, LiHRA members (see Box 1) were introduced to the 1047 concept by oral presentation at their annual meeting in 2016 and asked to submit questions in 1048 hard copy or by email; this request was repeated by email to the wider alliance membership.

1049 A total of 151 questions were submitted in this way from 17 members, all based in Europe.

1050 To broaden geographic inclusivity, members were asked to forward the link to a simple

1051 online survey through their international networks, which introduced the exercise and

1052 requested questions by free text entry. An oral presentation was also made at the $26^{\text {th }}$ biennial international conference of the World Association for the Advancement of Veterinary

1054 Parasitology (www.waavp.org), held in 2017 in Kuala Lumpur, Malaysia, and attended by $>500$ delegates from $>50$ countries, and again questions invited by completion of forms in

1056 hard copy on the day or by online survey. A further 28 questions from 9 people were

1057 submitted by hard copy, and 170 questions online from 32 people, following this exercise and an additional request at the LiHRA annual meeting in 2017. Finally, 36 questions were added from oral presentations at the WAAVP conference, having been identified by presenters as of

1060 pressing concern in their area of research. In total, 385 questions were submitted from at least 106158 people (excluding secondary sources and conference presenters). Participants were based

1062 in at least 19 different countries, widely distributed across Europe and also including 1063 Malaysia, South Africa, Pakistan, the USA, Canada, and New Zealand. Elicitation through more specific organisations and interest groups was avoided in case of bias; for example,

1065 soliciting questions through the EU COST Action COMBAR, which focuses on combatting 1066 anthelmintic resistance in Europe, might have preferentially raised questions on this issue. 
1067 The master list was reduced to 100 questions by online vote. Those who submitted questions, 1068 and the wider LiHRA membership, were asked to award each question zero, one, two or three 1069 stars, with more stars awarded to questions considered of high general importance and well 1070 suited to guide a focused and feasible research project or programme. The objective was to 1071 identify questions in important areas that are novel and testable, rather than those that are 1072 open-ended, general or already known. This choice was made using personal judgement, and 1073 there was no limit to the total number of stars that could be awarded by each voter. Question 1074 order was randomized for each participant. In total, 38 people voted, from a similar 1075 geographic profile as that of question submitters, comprising 15 countries, of which 11 in 1076 Europe, with many claiming direct experience of work in a wider range of locations spanning 1077 five continents.

1078 Questions were ranked according to total number of stars awarded, and in case of ties separated based on number of three-star scores awarded. When questions were repeated,

1080 effectively making the same point in a slightly different way, the highest scoring version was 1081 accepted, sometimes with minor changes to wording, others removed, and the next question 1082 on the list promoted into the top 100.

1083 A core group was constituted from those who engaged most vigorously with the process, and to cover the breadth of subject areas raised, as well as to bring perspectives from across the world. The core group made minor edits to questions, and then reached a consensus through

1086 written discussion on the split into ten topic areas, which represented major themes in the 1087 submitted list. The final list was presented in these sub-sections, with ranks removed.

1088 The methodology was adapted from earlier exercises in other subjects [10], modified to 1089 achieve greater global reach and less modification through repeated rounds of discussion. In 1090 this way, it was hoped that the final question list would capture a broad range of questions, 1091 unfiltered by expert opinion, relative to synthetic reviews. In the event, there was very little 

engagement from some parts of the world (e.g. Australia, South America) in spite of efforts to reach those regions, and a European bias in the core group and arguably therefore in the

1094 outcome, with a strong focus on anthelmintic resistance. The bias to Europe might be

1095 symptomatic of greater relevant research activity here than on other continents, but whatever

1096 the reason risks perpetuating focus on existing areas of strength in exactly the way this

1097 exercise sought to oppose. We exhort researchers in low and middle income countries in 1098 particular to seize the initiative in driving forward the research agenda to meet the needs in

1099 their countries, using researchers established elsewhere to support their efforts but not

1100 necessarily to determine the questions addressed or approaches used. It is also recommended

1101 that future elicitation exercises with similar aims make creative attempts to engage those who

1102 are less disposed to contribute, and further lessen the role of authors, for example by reducing

1103 the size and participation of the core group.

1104 
1106 Anthelmintic - a chemical which can be used to control worm infections. Six different broad-spectrum classes are currently widely available for use in sheep (benzimidazoles, imidazothiazoles, tetrahydropyrimidines, macrocyclic lactones, amino acetonitrile derivatives, and spiroindoles) and four for cattle (benzimidazoles, imidazothiazoles, tetrahydropyrimidines and macrocyclic lactones). The terms drug, wormer, and de-wormer are commonly used synonyms.

Anthelmintic resistance - the heritable reduction in the sensitivity of helminths to anthelmintics when animals have been administered the correct dose of the drug, in the correct manner, using drugs that are within date and have been stored correctly.

Animal Task Force (ATF) (www.animaltaskforce.eu) - a European public-private platform that fosters knowledge development and innovation for a sustainable and competitive livestock sector in Europe. See Box 1.

1118 Bioactive forages - crops or feedstuffs that reduce the numbers of worms in, or available to, a host. The effect can be either direct (anthelmintic activity; reduced survivability of freeliving stages on pasture) or indirect (improved nutrition).

Biological control - the control of infection using other organisms or their natural products, such as nematophagous fungi (Duddingtonia flagrans) or crystal (CRY) and cytolytic (CYT) proteins of the soil borne bacterium Bacillus thuringiensis.

DISCONTOOLS - www.discontools.eu is a publicly funded, open-access database to assist public and private funders of animal health research and researchers in identifying research gaps and planning future research. FAMACHA - FAffa MAllan CHArt -a colour-guide chart used to assess the degree of anaemia in an animal via the colour of their ocular membranes to determine the need for anthelmintic administration. Developed by three South African researchers (Drs Faffa Malan, Gareth Bath and Jan van Wyk) and named after one of the inventors.

Faecal Egg Count Reduction Test (FECRT) - a commonly used in vivo test to assess the efficacy of an anthelmintic through examination of egg counts of groups of animals pre- and post-anthelmintic administration. The reduction in faecal egg counts of treated animals is expressed as either a percentage reduction as compared to untreated control animals or using the treated animal as its own control (by comparing with the day-of-treatment count).

Host resilience - a host's ability to perform under parasite challenge.

Host resistance - a host's ability to control helminth infection, for example as illustrated by low worm burden or low faecal worm egg counts.

Hypobiosis - cessation in development of parasitic stages of roundworms within the host under unfavourable conditions, prior to resumption of development when conditions improve. Integrated parasite management (IPM) - the use of a combination of multiple control methods (chemotherapeutic and alternatives) to sustainably control helminth infections. Livestock Helminth Research Alliance (LiHRA) (www.lihra.eu) - a consortium of researchers that aims to develop sustainable effective helminth control strategies and promote their implementation by the livestock industry. See Box 1.

Plant secondary metabolites (PSM) - Plant products that are not directly involved in normal growth, development or reproduction, but instead are thought to be waste or stress products or defence mechanisms against herbivores and insects.

Refugia - parasite subpopulations from either the stages within the host or free-living stages that are not exposed to anthelmintic treatment, and that have the ability to complete their life cycle and pass on susceptible alleles to the next parasitic generation. This is generally achieved by ensuring that a proportion of the parasite population remains unexposed to drug, through either TT or TST (see below). 
1154 Star-IDAZ - International Research Consortium on animal health (www.star-idaz.net), 1155 comprising research funders and programme owners from Europe, Asia, Australasia, the 1156 Americas, Africa and the Middle East, as well as international organisations, and including 1157 representation from veterinary pharmaceutical companies. Members coordinate their research 1158 programmes to address agreed research needs, share results, and together seek new and

1159 improved animal health strategies for at least 30 priority diseases, infections or issues. See 1160 Box 1.

1161 Targeted selective treatment (TST) - the treatment of only some individual animals within 1162 a group at one time, instead of the more common whole-group treatment, where all animals 1163 in the group are treated simultaneously.

1164 Targeted treatment (TT) - treatment of animals at a time selected to either minimise the 1165 impact on the selection for anthelmintic resistance, or to maximise animal productivity.

1166 Zoonoses - infections that can be transferred from animals to humans.

1167 


\section{SUPPLEMENTAL MATERIAL}

1169 The full list of questions submitted, unedited, arranged in themes to reflect the manuscript.

\section{Helminth biology and epidemiology}

1. Are gastrointestinal nematodes transmitted from wild ruminants to domestic ones?

2. Are some species more or less pathogenic than they used to be?

3. Are there any new clinical techniques for the diagnosis of helminth infections of livestock?

4. Are there better ways of assessing parasite burden than WECs or weight gain?

1179 6. Can bio-marker detection system for helminths invasion detection be installed in milking

robot, so the farm manager will immediately get access to this information?

7. Can co-occurrence of other host species (e.g. wildlife) reduce anthelmintic resistance in livestock by introducing non-AR helminths?

8. Can farm management be included dynamically in models of helminth dynamics under climate change?

9. Can increasing the diversity of species present in an individual reduce disease from any single species?

10. Can we develop good ways to enumerate larvae on pasture?

11. Can we genetically modify populations of helminths to a less prolific and pathogenic form that would modify wild populations of helminths to become less pathogenic? 12. Can we improve understanding of future risks (eg. climate change and drug resistance)?

13. Can wildlife remove infective stages from the environment and hence decrease parasite infection pressure for livestock?

14. Can you link parasite population dynamics to parasite population genetic structures, and subsequently to variability in parasite pathogenicity and life-history traits?

15. Do bio-markers in milk or saliva of livestock for early detection of helminth invasion that needs to be treated exist?

16. Do different species of GIN have different levels of impact?

17. Does a compatibility filter (as defined by Claude Combes) exist in terms of genome interaction between the parasite and the host? 18. Does AR affect helminth life histories outside of hosts?

19. Does cross-grazing of cattle and sheep encourage GI nematode species to adapt and cross between hosts?

20. Give three reasons why infections with helminths are still very important in livestock?

21. Have parasites with relatively long life-cycles been selected for shorter life cycles by frequent use of anthelmintics, as a parallel but independent selection process distinct from selection for drug resistance?

22. How are incoming Ascaridia galli larvae affected by either mucosal phase larvae and/or adult worms?

23. How are parasites evolving to deal with recent movement into climates very different from where they evolved over millions of years?

24. How can advances in parasite control be extended to less wealthy countries?

25 . How can advancing high throughput technologies offers the prospect of progress in the area of applied parasitology? 26. How can free-living nematode stages survive on pastures? 27. How can helminths be managed on small farms with minimal grazing land? 
28. How can we better practically detect and quantify viable liver fluke stages on pasture?

29. How can we better practically detect and species ID/profile GIN larvae on pasture? 30 . How can we define the key features of new anthelmintics, taking into account user and environmental safety?

31. How can we effectively combine pasture management and parasite risk software?

32. How do free living stages of nematodes adapt to climate change?

33. How do infections with intestinal helminths affect the growth of young animals?

34. How do parasitic worms respond to climatic change and what is the environmental plasticity?

35. How do the different species of parasite present in an individual interact?

36. How do water management and grazing practices interact to determine infection rate with Schistosoma species in ruminants?

37. How does climatic change affect parasitism in grazing animals especially in semi-arid areas?

38. How harmful are tapeworms to sheep and goats?

39. How is climate change affecting overwintering of nematodes in temperate areas?

40. How is hypobiosis from ruminant GIN terminated?

41. How may massive anthelmintic chemotherapy in animal farming alter the life-traits of parasites?

42. How to control helminthiasis among small ruminants?

43. In co-grazing systems how often do cattle carry Haemonchus contortus and what are the consequences (biological and on weight gain or production)?

44. Is Dicrocoelium dendriticum a parasite worth combatting?

45. Is Haemonchus dominance really spreading in temperate areas and what difference should it make to worm control advice?

46. Is the epidemiology of lungworm (Dictyocaulus viviparus) changing - why so many outbreaks in older (dairy) animals?

47. Is the eradication of Taenia solium feasible?

48. Is the recent prevalence increase of rumen fluke in Europe a threat to livestock farming?

49. Should we really aim to eliminate GIN in grazing animals or had we better sustain them?

50. To what extent are we dealing with neglected parasites when we are examining faecal samples?

51. To what extent is extreme adaptation is considered genetic drift/shift in helminths?

52. To what extent is there an exchange of parasites between wild and domestic ruminants?

53. What are the dynamics of resumption of development of inhibited larvae in horses (cyathostomes)?

54. What are the emerging issues/diseases in helminthology?

55 . What are the functional roles of genomic 'non-coding' dark matter?

56. What are the longitudinal infection dynamics of Dictyocaulus viviparus within a herd of supposedly immune cattle over a number of subsequent years?

57. What are the major factors affecting infection levels of grazing animals with helminths?

58. What are the major genomic changes that enable species to adapt to a warmer climate?

59. What are the paramount parameters to assess the morbidity due to helminth infections? 
60. What are valid grounds on which to separate parasite species?

1269 61. What do we understand about geographical differences and genetic variation in parasite 1270 populations?

1271 62. What is the balance between drift and selection in gastro-intestinal nematode evolution?

63. What is the cause of the reduction in voluntary feed intake in parasitized animals?

64. What is the clinical relevance of AR in e.g. sheep or horses?

65. What is the demonstrable effect of climate change on helminth parasites of livestock (+ve or $-\mathrm{ve})$ ?

1277 66. What is the difference in pathogenesis, effect on production, distribution and AR status

1278 between Cooperia punctata, C. pectinata and C. oncophora?

1279 67. What is the effect of helminth infection on GHG emissions from livestock, either directly

1280 or indirectly? 68. What is the effect of weather/climate (especially drought) on the spatial distribution of GIN infective larvae on pasture and on the subsequent parasitical risk for grazing animals?

69. What is the efficient size of populations in gastrointestinal nematodes?

70. What is the empirical evidence that different parasites will respond on global climate change?

71. What is the epidemiology of $H$. contortus in northern Europe?

72. What is the genetic basis behind hypobiosis?

1289 73. What is the impact of helminth parasitism in Europe in 2017 ?

1290 74. What is the influence of global change in the dynamics of the epidemiology of GIN?

1291 75. What is the inherent ability of a nematode to modulate its life-history traits to adapt to

1292 environmental pressures?

1293 76. What is the pathogenic effect of rumen fluke?

1294 77. What is the potential for parasite genomes? How should we use the information and what 1295 will they yield?

1296 78. What is the prevalence of various helminthoses?

1297 79. What is the relationship between parasitic diseases and the main infectious diseases of 1298 livestock?

1299 80. What is the relevance of the wild animal - domestic animal interphase for the main 1300 parasitic diseases of livestock?

1301 81. What is the role of wildlife in disseminating livestock parasites \& AR

82. What is the spatial distribution of helminth infections and how are they interrelated? 83. What is the impact of anthelmintics on non-target fauna, functioning and ecosystem service provision?

84. What percentage of adult dairy and beef cattle carry worms or lesions from Ostertagia and what effect does this have on production?

85. When identifying wildlife reservoirs how much focus is put on identifying the direction of parasite transfer?

86. Where did Calicophoron daubneyi come from?

87. Which factors determine the length of the mucosal phase of Ascaridia galli?

88. Which helminth is more affected by climate change? Is it temperate or tropical? Why?

89. Which parasites will be the winners and losers according to climate change models?

90. Which user-friendly input data are required on a farm level to get useful output from a decision support tool or a transmission model?

91. Why do horses lack important groupings of parasites that are common in other grazing ungulates? 
1318 92. Will climate change result in a change of species in temperate environments or will the

1319 existing ones simply adapt?

1320 93. What regulates egg production in females and can we suppress female egg production sufficiently to provide an epidemiological advantage?

94. Will breeding for resistance (low FECs and high production potential) drive nematode adaptation towards increased fecundity to compensate?

\section{Helminth biology and epidemiology - diagnostics}

95. How can I see or detect that my flock or herd is infected by helminths?

96. How can we improve the diagnosis of Fasciola spp?

97. How far are we away from tests in the live animal for immature fluke and Nematodirus infestations?

98. How to predict a clinical case of dictyocaulosis in cattle?

99. In a flock or herd, which sampling protocol should be followed for the diagnosis of helminth infections?

100. Is a mixed species of GINs in one animal difficult to control compared to an infected animal with one GIN species?

101. Is there some general European strategy for (manual) of examination of livestock for helminthoses, before a treatment? Which methods are used in particular countries? 102. What new technologies are used to detect infections by helminths in livestock? 103. When will automated diagnostic tools/technologies be really available for on-farm diagnosis?

104. Which user-friendly parameters can help the farmer (or veterinarian) to make informed decisions on helminth control in young stock?

105. Why are faecal egg counts not at all times a good parameter to assess worm counts of strongyles?

\section{Economic and environmental impacts}

106. From an economical and ecological point of view, what helminths do farmers think are the most important? How would they list them?

107. How accurately can we predict changes in the seasonality and magnitude of risk? 108. How can helminth control be integrated in farm management in a cost-efficient way?

109. How can we better assess production and health impacts of helminths?

110. How can you measure environmental impacts of anthelmintics?

111. Can we put an economic dollar value on the importance of a more strategic approach to GIN treatment to producers?

112. How does helminth control impact on the environment (MLs on microorganisms, environmental schemes etc)?

113. How important is it for us to chase subclinical GI nematodes in grazing beef cattle with low FEC?

114. How the three main farming systems (capitalistic, entrepreneur-type, peasant / small farming / family farming) modify through values and technicity the parasite community? 115. Is profitable livestock husbandry possible without chemical parasite control? 116. Is there a market space to promote livestock products raised without (or with limited) use of anthelmintics?

117. Is there an association between countries or regions that have high levels of Fasciola and level of income in those countries / regions?

118. Is there an impact in the environment by the overuse of anthelmintics over the past decades? 
1367 119. Should we be advising anthelmintic treatment of dairy cows with antibodies to $O$.

1368 ostertagi but no clinical signs? Is a potential $1 \mathrm{~kg} / \mathrm{d}$ increase in yield worth the cost, time and

1369 increased use of anthelmintics?

1370 120. What are the consequences on productions of helminth infections (including pigs and

1371 poultry)?

1372 121. What are the costs (financial, human and welfare) of anthelmintic resistance?

1373 122. What are the economics of GIN and Fasciola infection in cattle?

1374 123. What are the long-term impacts of anthelmintics on beneficial dung fauna and their

1375 functioning?

1376 124. What is the economic burden of helminths of livestock in each country around the

1377 world, in 2017?

1378 125. What is the economic impact of anthelmintic resistance in livestock?

1379 126. What is the economical impact of strongyle infections in ruminants?

1380 127. What is the real impact of parasitic gastroenteritis on small ruminant production?

1381 128. What is the true financial cost of helminth infection?

1382 129. What is the true on farm economic impact of sheep (and cattle?) bred for resistance and

1383 is it a viable option for future breeding? E.g. impact on reducing pasture contamination /

1384 subsequent parasite challenge?

1385 130. Which factors determine the role of helminth infections in the whole-farm economic

1386 context?

1387 131. Will the benefits of helminth control of livestock for global environmental sustainability

1388 become as important as economic benefits are now when promoting our research?

1389 132. Does the control of helminths reduce the net methane emission over the lifetime of a ruminant?

\section{Effects on host behaviour and welfare}

133. Are animals better off and healthier with some worms, rather than none?

134. Can we select for host behaviour to control helminth infections?

135. Do ruminant parasites change the behaviour of the host?

136. Do ruminants graze complex vegetation selectively to avoid nematode infection? 137. Do ruminants self-medicate by selectively grazing plants with anthelmintic compounds?

138. How can parasites be beneficial to hosts (individually or in terms of population or species levels)? All studies are biased on the negative effect on host.

139. How can we develop animal production supportive and welfare based control strategies in soil-transmitted helminth infections?

140. How does parasitism affect animal behaviour and can we use changes in behaviour as a way of identifying those that need treatment?

141. How can we measure the impact of helminth infections on livestock welfare?

\section{Host-helminth-microbiome interactions}

142. Are there associations between animals' microbiomes and helminth communities? parasitic disease in livestock?

146. How is the pathobiome considered in the host genetic selection scheme?

147. How strong is the influence of microbiota on nematode diversity? 148. What is the importance of climate change, helminth infections and immune response to inter-current microbial infectious diseases? 
1417 149. How do co-infections with helminths, and other infective organisms influence impact on

1418 each other by direct or indirect immunologically related effects?

1419 150. What is the role of co-infections e.g. bTB \& fluke; ParaTB \& GIN etc.?

1420 151. What is the role of GIN in modifying the gut and lung microbiomes, and how does this

1421 impact risk of bovine respiratory disease?

1422 152. How do host-parasite relationships evolve when the initial conditions are nearly (but not fully) the same: an application of the deterministic chaos of Poincaré? 153. How do GIN communicate in the GI tract? 154. How does interaction between different helminth species in co-infection affect the immune system of the host?

\section{Host resistance / resilience and selective breeding}

155. Are there any advantages to being an individual that is prone to high parasite burdens?

156. Breeding for resilience (high FECs and high production potential) could result in significantly increased pasture contamination over many years. What will the impact of higher challenges be on resilient individuals? Will the resilience break down above a certain threshold?

157. Can use of resilient sheep in a 'normal' flock (no Haemonchus) act as a source of susceptible nematodes?

158. Has 60 years of intense anthelmintic use changed the relative susceptibility of livestock to parasites? In other words, are animals wimpier than they used to be as a result of protection from the effects of parasites by drugs, thereby causing selection of higher producing but more parasite-susceptible animals?

159. How can genetic/gene manipulation be used in the parasite or the host to help with the control of helminths?

160. To what extent is the impact of strongylid infections in ruminants dependent on host resilience?

161. Under what circumstances should breeders aim for resilience, versus resistance, in livestock?

162. What impact will breeding of sheep for resistance and resilience to nematodes have on nematode challenge and adaptation?

163. Which are the main differences between cattle, sheep and goats in term of resistance/susceptibility to helminth infection?

164. Which genotypes of livestock hold natural resistance to helminths?

165. What do we understand about the fitness cost of resistance and how can it be measured? 166. Why are some animals more prone to heavy parasite burdens than others?

167. How to measure and distinguish the resilience and the resistance of ruminants infected with GIN?

168. Is resistance or tolerance a better breeding objective to produce small ruminants that require less anthelmintic treatment?

169. Can targeted selective treatment, e.g. using FAMACHA, be used to select for parasite resilience, especially among low-input traditional breeds?

170. In non-selective breeding systems, does TST support weak animals and lead to loss of resilience at herd or flock level?

171. What are the life-time trade-offs between immunity to helminths and impacts on growth and production, in different livestock systems?

\section{Development and detection of anthelmintic resistance}


1466 172. Are data on drug failure/drug resistance within countries publicly available and are they

1467 reliable enough to be used as a mechanism to survey drug failure/resistance at a national /

1468 international level?

1469 173. Are data related to helminth resistance available for particular European countries?

1470 174. Can the use of combination drugs help to slow down the development of anthelmintic

1471 resistance?

1472 175. Can we develop markers for susceptibility to ML anthelmintics?

1473 176. Can we improve methods for monitoring efficacy of current control methods (e.g.

1474 surveillance, diagnostics and resistance testing)?

1475 177. Can we replace worm egg counts with an on-farm 'colour-change', e.g. ELISA,

1476 technology?

1477 178. Do combinatorial effects of different resistance mechanisms (i.e. target-associated and

1478 non-target-associated) exist and if so to what effect is this relevant in the field?

1479 179. Do differences in life history traits and reproductive strategy affect the risk for

1480 development of anthelmintic resistance?

1481 180. Do intra-ruminal bolus systems have an impact on the development of anthelmintic

1482 resistance?

1483 181. Does copy number variation have a role in anthelmintic resistance?

1484 182. Does gene duplication play a role in anthelmintic resistance?

1485 183. Does selection by ivermectin preselect for moxidectin resistance?

1486 184. Has the selection for drug resistance changed the pathogenicity of parasites?

1487 185. How can the knowledge on AR in livestock be used to promote a better understanding of

1488 the development and mechanisms of AR in human GIN?

1489 186. How can we design anthelmintic combinations that are more effective and that

1490 should/would limit resistance development?

1491 187. How can we develop molecular markers for ML drugs?

1492 188. How can we improve diagnostics: infection intensities and drug resistance?

1493 189. How do we prevent anthelmintic resistance, when change makes it a moving target?

1494 190. How does animal movement affect the spread of helminth infections and anthelmintic

1495 resistance?

1496 191. How fast is AR developing in cattle nematodes?

1497 192. How is size of refugia needed affected by the genetics of ML resistance?

1498 193. How predictive can be a gastro-intestinal nematode model in terms of resistance

1499 appearance and emergence?

1500 194. How useful are composite faecal egg counts to detect anthelmintic resistance?

1501 195. In-vitro/genetic/lab methods for detection of anthelmintic resistances: desirable,

1502 reachable and applicable for all anthelmintic drug groups?

1503 196. Is there evidence of selection for ML-R when treating for sheep scab?

1504 197. Is treatment of ectoparasites with macrocyclic lactone drugs an important driver of

1505 anthelmintic resistance in sheep?

1506 198. Practically, what should the percentage of sheep/goats/cows/heifers left untreated in a

1507 group to control the emergence of anthelmintic resistance?

1508 199. What are the best diagnostic techniques to detect anthelmintic resistance?

1509 200. What are the contributory factors for the development of anthelmintic resistance?

1510 201. What are the key factors involved in the development of AH resistance, and mitigation

1511 measures?

1512 202. What are the molecular mechanisms involved in resistance to macrocyclic lactones?

1513 203. What are the prospects for identifying molecular markers for resistance?

1514 204. What are the risk factors for multiple anthelmintic resistance development in cattle? 
1515 205. What changes in genes other than the immediate drug target, such as transporters and

1516 drug metabolism are involved in drug resistance?

1517 206. What do genotype-phenotype studies tell us about the quantitative contribution of a

1518 particular mutation to the resistance phenotype?

1519 207. What do we learn from the virtual absence of anthelmintic resistance in cattle?

1520 208. What drugs are the cause of higher prevalence of anthelmintic resistance in cattle, sheep

1521 and goats?

1522 209. What factors are involved in the development of anthelmintic resistance?

1523 210. What factors drive the emergence of anthelmintic resistance?

1524 211. What is the best way for in vivo quantitative evaluation of GIN burden in cattle?

1525 212. What is the effect of long lasting moxidection injections of the development of ML

1526 resistance in sheep and cattle?

1527 213. What is the empirical evidence for a lack of reversion to susceptibility when drug

1528 selection pressure is removed?

1529 214. What is the global scenario of prevalence and optimal methods for detection of

1530 anthelmintic resistance in ruminants?

1531215 . What is the key to molecular assays capable of detecting resistant worms?

1532 216. What is the link between genetic variation and the risk for selection of resistance?

1533 217. What is the relative importance of management versus environmental factors in

1534 determining the development of anthelmintic resistance in livestock?

1535 218. What is the role of combination i.e. dual-active anthelmintics in current helminth

1536 control?

1537 219. What is the role of sequencing (WGS/NGS) in understanding the genetic basis of AR in

1538 GIN \& fluke? 220. What is the status of drug resistance in Ascaris suum and other important pig parasites?

221. What is the true, non-biased, prevalence of anthelmintic resistance?

222. What makes a parasite resistant to anthelmintics?

223. What role does the individual animal play in the development of drug resistance in a parasite population?

224. What specific genetic differences either cause resistance or are sufficiently closely associated with resistance to be able to serve as molecular markers?

225. Where are we at present in anthelmintic resistance in farm animals?

226. Which are the most rapid and accurate methods to detect the anthelmintic resistance?

227. Which are the newest anthelmintics available in the market, and is there any report about flock or herds resistant to these ones?

228. Which genes are implicated in the development of anthelmintic resistance according to the family of anthelmintic?

229. Why did AR (at least thus far) not occur in most gastro-intestinal helminths of dogs and cats?

230. Why is it so difficult to identify markers for genetic resistance?

231. Is there (genetic) evidence for reversion to susceptibility under any circumstances?

\section{Practical management of anthelmintic resistance}

232. Anthelmintic treatment and control programmes: where, who, when and how?

233. Are combination anthelmintics useful to combat anthelmintic resistance?

234. Are current control programmes suitable for helminths in livestock considering all or most of the productivity systems? 
1564 235. Can 'farmer's eye be used effectively to slow the development of AR in sheep flocks (it

1565 works but what about its effect on performance)?

1566 236. Can we expect new anthelmintic compounds on the market in the (near) future?

1567 237. How much are the major pharmaceutical companies investing in new anthelmintics,

1568 specifically?

1569 238. We are on the cusp of having molecular markers for drug resistance e.g macrocylic

1570 lactone resistance in Haemonchus contortus and triclabendazole resistance in liver fluke.

1571 How should we best apply these markers?

1572 239. Should focus on new drug discovery ensure the target is just one class of parasite so that

1573 resistance development due to inadvertent use can be minimised? E.g. if an injectable

1574 treatment for external parasites such as scab can be developed which doesn't also control

1575 roundworms.

1576 240. What are the limitations for developing anthelmintic combinations?

1577 241. What are the prospects for a new flukicide to treat immature/acute infection, especially

1578 in sheep?

1579 242. What are the prospects for any novel anthelmintics, given experiences with new AADs

1580 \& dual-actives?

1581 243. What is the value of faecal egg count monitoring as a decision tool in anthelmintic

1582 treatments?

1583 244. Is TST a feasible approach with which to control helminths with a very high biotic

1584 potential, e.g. the ascarids?

1585 245. What reporting systems are in place to record drug failure/drug resistance within countries?

246. Could an anthelmintic-resistant flock or herd get back to be susceptible and how?

247. Describe the methods of integrated helminth parasite control?

248. Can we automate TST data interpretation, also for farmer training?

249. How can flukicides be applied more effectively, is refugia an option?

250. How can we make control more effective and sustainable?

251. How do we apply existing knowledge of the risk factors for anthelmintic resistance on farms to effectively slow its development?

252. How can we reverse AH resistance?

253. How do we implement better dosing procedures of anthelmintics to cattle in order to insure therapeutic drug levels (pour-on vs. injection/oral)?

254. How do we solve the conundrum of use of anthelmintic drug combinations - or when to use drug combinations and when not to?

255. How does the level of refugia influence the emergence of resistant phenotypes?

256. How to control anthelmintic resistance?

257. Is anthelmintic resistance genuinely irreversible or can susceptibility be restored within helminth populations?

258. Is deworming sheep or goats truly necessary?

259. Under what circumstances are combination drugs the answer to manage anthelmintic resistance?

260. What (empirical) evidence is there that refugia slows down the development of drug resistance?

261. What are the best strategies to prevent further spread of anthelmintic resistance (in small ruminants)?

262. What are the characteristics of an optimal quarantine drench as a way of reducing the risk of importing resistance with bought in animals?

263. What is the efficacy of mitigation measures to reduce non-target impacts of anthelmintic on the environment? 
1614 264. What is the optimal use of fasciolicides where there is triclabendazole resistance?

1615 265. What is the role of refugia in slowing selection for AR in sheep/cattle GIN?

1616 266. What is the usefulness of anthelmintics working at decreased (50\% or $80 \%)$ efficacy?

267. What proportion of a parasite population must be left in refugia?

1619 268. What steps should be taken when resistance to all known anthelmintic drug classes

1620 develops? 269. Is refugia relevant for all parasite species; if not, what realistic alternatives exist for those parasites that display drug resistance but for which refugia based control is not deemed appropriate? 270. What will be the best methods to control Fasciola in areas where there is free grazing?

271. Why is development of anthelmintic resistance not reversible, even in the absence of the specific drug?

272. Is targeted selective treatment sustainable in the long term?

273. Why is the (parasitological) community accepting strategic anthelmintic treatments against GIN in cows (not learning from the small ruminant example?

274. With good parasite management can on farm anthelmintic resistance be reversed? Especially to $2 \mathrm{LV}$ and $3 \mathrm{ML}$ classes of drugs as has been found in NZ? 275. Is there a link between the size of the refugia needed to prevent AR and the molecule used (persistent versus non persistent)? 276. How does the level of refugia influence the detection and spread of resistant phenotype in different hosts, different parasites and different treatment systems? 277. Is there a role for refugia in control of liver fluke? 278. What are the most useful decision parameters in targeted selective anthelmintic treatments?

\section{Vaccines and immunology}

279. Can we develop sustainable methods of control (eg. vaccines and management)? 280. Can we enhance the natural immune response to helminths by applying a biological treatment (e.g. specific cytokine or cytokine inhibitor) and thereby control them effectively?

281. Could immune-stimulatory drugs for livestock be used for combating helminths? 282. Does Fasciola modulate co-infection with other parasites?

283. Do worms have a microbiome? Can it be exploited as a vaccine or treatment target? 284. How are optimal helminth vaccination schedules influenced by infection pressure and can this be incorporated into decision making?

285. How can vaccines against helminth infections in ruminants be integrated in control programmes?

286. How can we develop and apply vaccines?

287. How does the parasite resist or escape from the host immune system?

288. How fast do parasites adapt to increased immune selection pressures (due to for instance vaccines)?

289. How may massive anthelmintic chemotherapy in animal farming alter host immunity structuration?

290. How well do anti-helminth vaccines have to work to be useful?

291. How would vaccines against soil-transmitted helminth infections influence population dynamics?

292. To what extent does overuse of/use of very effective anthelmintic products affect development of immunity to bovine lungworm? 
1664 293. To what extent is the immunomodulation by helminth parasites detrimental to the

1665 animal's health when co-infections co-occur?

1666 294. What are the crucial effects that a vaccine against helminth(s) need to produce so that

1667 farmers agree to include them in their farm management?

1668 295. What is the future for (recombinant) vaccines?

1669 296. What is the future of vaccines against helminths of livestock?

1670 297. What is the immunological difference between host species showing widely different

1671 responses to closely related parasite species (eg. cattle versus donkey with respect to

1672 Dictyocaulus spp.)?

1673 298. What is the potential for a multivalent vaccine to control multiple species?

1674 299. What is the potential for vaccines to control individual helminth species?

1675 300. What mechanisms are involved in protective immunity against helminths?

1676 301. What regulates egg production in females and can we suppress female egg production

1677 sufficiently to provide an epidemiological advantage?

1678 302. Which efficacy is needed from a helminth vaccine and how can vaccination be

1679 integrated in sustainable parasite control?

1680 303. Why don't we yet have vaccines to control helminth infections in livestock?

1681 304. Why is the efficacy of the Haemonchus vaccine (hidden antigen approach) much lower 1682 in adult sheep?

1683 305. Why is the protective immunity to Ascaridia galli limited or almost absent?

\section{Alternative approaches to helminth management}

306. Are there basic processes in egg hatching or larval development that can be manipulated to aid control?

307. Are there possible escaping mechanisms of GIN to alternative approaches (e.g. vaccines, bioactive compounds)?

308. As challenge increases, will this result in an increase in the proportion of the flock/herd needing treatment over time?

309. Can anthelmintic resistance be reversed through TST, good management or reseeding approaches?

310. Can different bioactive plants be combined to increase effects on GI nematodes?

311. Can knowledge of risk factors for nematode infection in cattle, derived from antibody testing, be used to target treatments more effectively within as well as between herds?

312. Can TSTs be applied to cattle or pig parasites?

313. Can we cultivate plants for grazing which have maximum nutritive value and the potential to lower helminth burden?

314. Can we manipulate the intermediate host (e.g. Galba truncatula) to help control Fasciola hepatica and Calicophoron daubneyi?

315. Can we use polyphenols or other natural compounds found in forage to control helminths of livestock?

316. Does a natural polyphenol causing $100 \%$ inhibition of L3 of GIN larvae in vitro represent a promising natural compound for integrated helminths control??

317. Does feeding of probiotics improve resistance to and outcome of GI helminth infection?

318. Does the inhibition of exsheathment of L3 stage of gastrointestinal nematodes represent a viable control method for these helminths?

319. How can investigation of tank milk be an attractive monitoring tool so that it can be used as a basis for intervention strategies? 320. How do we develop easy, on-farm tools (diagnosis) for the implementation of targeted selected treatments? 
1714 321. How does processing and conservation of bioactive forages affect their efficacy?

1715 322. How is the pharmacokinetic behaviour of bioactive plant compounds in relation to

1716 parasitic nematodes situated in different body compartments (i.e. small intestine, large

1717 intestine, liver, lungs)?

1718 323. How should vaccines be combined with anthelmintics to optimise control?

1719 324. How successful are herbs as an alternative of anthelmintic to livestock helminth?

1720 325. If reduced effectiveness of TST over time transpires, could targeted treatment instead of

1721 TST be used to minimise pasture contamination at strategic intervals e.g. every few years at a

1722 time of year when egg development success is greatest?

1723 326. Is on-farm TST applicable in cattle viz-a-viz FAMACHA in sheep?

1724 327. How can we practically target free-living gastrointestinal nematode stages outside the

1725 host?

1726 328. Is TT (treating at times of highest risk) inherently incompatible with the aim of

1727 maximising refugia? E.g. by treating at the time when risk is highest (usually when

1728 development success is high) we are increasing the selection pressure.

1729 329. Many studies have shown a maximum efficacy of bioactive (plant) compounds around

$173060-70 \%$ reduction - how do we get a higher efficacy? Is it needed?

1731 330. Should TST be adapted to overall infection levels, such that whole-herd treatments are

1732 sometimes optimal?

1733 331. To what extent should TST indicators for nematode infection be extended to include

1734 arthropod parasites?

1735 332. What are the alternatives to anthelmintic drugs?

1736 333. What are the interactions between bioactive forages and synthetic anthelmintic drugs, in

$1737 \quad$ vitro and in vivo?

1738 334. How successful are herbs as an alternative of anthelmintic to livestock helminth?

1739335. What are the limitations of pasture management routines?

1740 336. What are the mechanism of action of bioactive plant compounds and metabolites in

1741 relation to parasite establishment and adult worms?

1742 337. What is effective worm control within a context of sustainability?

1743 338. What is the best alternative to anthelmintics?

1744 339. What is the effect of the use of alternative control measures (i.e. bioactive plants) as

1745 regards $\mathrm{AH}$ resistance?

1746 340. What is the efficacy of alternative methods of livestock parasite control?

1747 341. What is the efficacy of dung beetles for livestock helminth control?

1748 342. What is the role of medicinal plants for developing new anthelmintics?

1749 343. What should be the minimal size of a refugia population to ensure the efficacy of a TST

1750 strategy to prevent AR in ruminants?

1751 344. Why does the Duddingtonia (BC) approach work less well in small ruminants?

1752 345. Will TST result in increased pasture contamination over many years? Especially with

1753 increased overwinter survival of L3 on pasture.

1754 346. What is the efficacy of plant based anthelmintics against drug resistant helminths?

1755 347. What are the main obstacles to the development of new technologies to control

1756 helminths of livestock?

\section{Stakeholder engagement}

348. Are farmers able to adapt or do they need support (e.g. from predictive models)? Does this vary by sector e.g. dairy vs sheep?

349. Are farmers and/or vets from rural regions being well advised on what are the best practices for parasite control in their area?

350. Are our models any better than farmers' intuition? 
351. Can veterinary surgeons get more involved in parasite control on sheep farms?

352. Can we convince producers to adopt more sustainable control practices (where resistance is not yet an issue; to prevent its development)?

353. How can different novel control methods for GI nematodes be integrated effectively and in a way that is simple enough for farmers to implement?

354. How can famer perceptions of anthelmintic resistance as something that happens to others be overcome to increase their efforts to combat it?

355. How can we better promote best practices of diagnosis and treatment for helminth control in livestock?

356. How can we improve uptake of sustainable parasite control measures by vets and farmers?

357. How can we increase correct management against parasitoses by livestock farmers? 358. How can we refine spatial granularity of farmers' data whilst protecting privacy?

359. How do we (the vet parasitology research community) achieve recognition for scientific papers that are aimed at practitioners, who do not publish themselves and therefore add nothing to citation rates?

360. Can we be more creative in delivering alternative control options to farmers, including in less developed countries?

361. How do we communicate the importance of a more strategic approach to GIN treatment to producers? Can we put an economic dollar value on it?

362. How does the attitude of farmers with respect to accepting and implementing parasite control measures differ between countries?

363. How sustainability are farmer out-reach projects on helminths?

364. How to improve the relationships (eg submission of shared projects) between Vet and Medical Helminthology (Parasitology)?

365. How will consumers influence livestock production practices, in terms of anthelmintic use?

366. How will farmers adapt to the impact of climate change (increased climate variability) on disease risk?

367. If tools were available to support farmers, what is the best way to encourage their use? Demonstration farms etc.?

368. In which direction can we improve evidence based medicine for helminth control by dairy veterinarians?

369. Is research in veterinary helminth infections reaching livestock farmers in developing countries and, if so, what is the impact?

370. Is the stronger regulation of the sale of anthelmintics the only current way to slow the continued development of anthelmintic resistance?

371. Vets, farmers, pharmaceuticals, researchers, stakeholders: which role for each one in the integrated control of parasites?

372. What are the treatment approaches currently applied by producers?

373. What factors drive anthelmintic treatment decisions by farmers?

374. What is the optimal way to deliver spatial decision support to farmers?

375. What is the role of human behaviour and psychology on livestock diseases?

376. What kind of practice from the farmer would help to get livestock free of helminths?

377. Why do most trust more on chemical parasite control than on adapting animal husbandry and grazing based on parasite life cycles?

378. Why does farmer uptake of crucially important recommendations fail? 379. Why we have been failing to achieve an integrated and sustainable helminth control programme? 
1814 380. Can we integrate helminth control decision support tools in farm management software?

1815 381. How can we transfer automated technology to farmers, especially those that are

1816 resource-poor?

1817 382. What can we learn from social sciences to transfer knowledge on helminth control to

1818 farmers?

\section{Others}

1821 383. How can we best protect parasitology as a distinct discipline in 'systems-based'

1822 veterinary school curricula?

1823 384. How do helminths infections in livestock impact stunting rates in children of subsistence 1824 farmers?

1825 385. What is a helminth parasite?

1826 386. What is the better way to fight these pests?

1827 387. What is the effect of parasite control programmes on product quality and safety?

1828 388. What is the European general treatment strategy of treatment of helminths in livestock?

1829 Which chemotherapeutics are used in particular countries? 